THE PLANNING AND CONTROL OF MANUAL AIMING MOVEMENTS 


\title{
THE EFFECT OF THE MÜLLER-LYER ILLUSION ON THE
}

PLANNING AND CONTROL OF MANUAL AIMING MOVEMENTS

\author{
By
}

JOCELYN MENDOZA, H.B.KIN.

\author{
A Thesis \\ Submitted to the School of Graduate Studies \\ in Partial Fulfillment of the Requirements \\ for the Degree of Master of Science \\ McMaster University \\ C Copyright by Jocelyn Mendoza, June 2004
}


MASTER OF SCIENCE (2004)

(Kinesiology)
McMaster University

Hamilton, Ontario

TITLE: The Effect of the Müller-Lyer Illusion on the Planning and Control of Manual Aiming Movements

AUTHOR: Jocelyn Mendoza, H.B.Kin. (McMaster University)

SUPERVISOR: Professor Digby Elliott, Ph.D.

NUMBER. OF PAGES: vii, 99 


\begin{abstract}
Three experiments were conducted to investigate the two visual systems hypothesis (Milner \& Goodale, 1995) and the planning-control hypothesis (Glover, 2002).

Experiment 1 required the participants to make rapid aiming movements to $25 \mathrm{~cm}$ and 35 $\mathrm{cm}$ tails-in, no tails, or tails-out Müller-Lyer stimuli following a $0 \mathrm{~ms}$ or $5000 \mathrm{~ms}$ novision delay. In Experiment 2, the participants executed their movements with full vision of the Müller-Lyer vertices that either remained the same or changed to a different configuration upon movement initiation. Vision was occluded either $350 \mathrm{~ms}$ or $450 \mathrm{~ms}$ after the onset of the movement. Experiment 3 was similar to Experiment 2, except the amount of visual feedback for on-line control was constrained to $200 \mathrm{~ms}, 400 \mathrm{~ms}$, or 600 ms. The results of these experiments are problematic for both hypotheses. The participants exhibited a similar pattern of illusion-induced bias in both short and long delay conditions. In addition, the magnitude of the aiming bias increased as the moveme:t unfolded (Experiment 1). Furthermore, even though participants were engaging; in on-line control the illusion continued to exert its effects on aiming during the latter stages of the movement (Experiment 2). This effect was also observed when participents had sufficient time to process visual feedback in order to modify their movements (Experiment 3). Taken together, the results suggest that on-line control is biased by visual illusions.
\end{abstract}




\section{ACKNOWLEDGEMENTS}

First and foremost, I would like to thank Dig for all of his support in the past 2 years. I hope this thesis makes you proud; this thesis is what it is because of you. I would also like to thank my committee members, Jim, Dan, and Tim for their insightful input. Thanks Dan for traveling from Guelph, I greatly appreciate it! I would also like to thank my family for always being there for me, and for inspiring me day in and day out.- You have taught me that hard work and perseverance all pays off in the end! Thanks to Missy (my cat) for listening to me ramble on about random things... although you never say anything in return, you comfort me when I need it the most! For all of my friends in the lab, you have kept me grounded and sane when I was on the verge of losing it. Special thanks go out to Vicky, Courtney, Clare, Kate, Steve, Jae, and Cheryl for being there when I needed you. I will always remember the times we shared together! Finally, I would like to thank Daniel Laine for everything. Your never-ending support has gotten me through these years. I cannot thank you enough for listening, instilling confidence in me when I felt I didn't have anything left, and for encouraging me to push forward. Your love means the world to me. 


\section{TABLE OF CONTENTS}

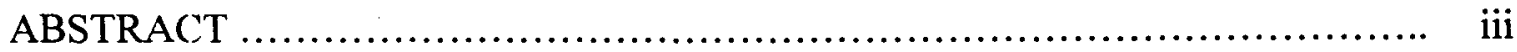

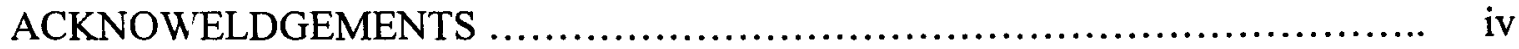

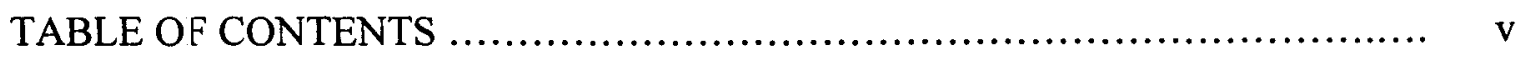

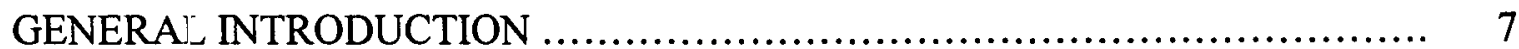

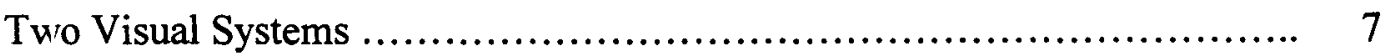

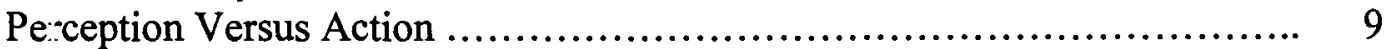

Perception Influences Action . ........................................ 14

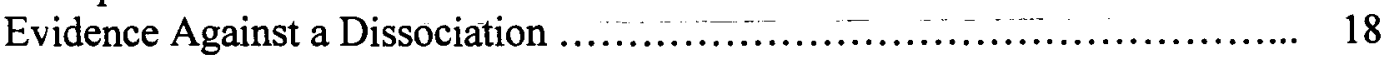

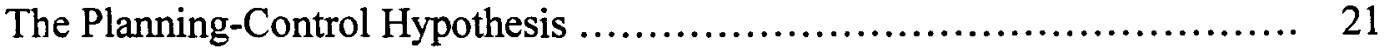

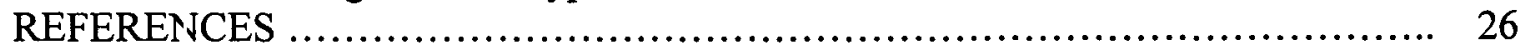

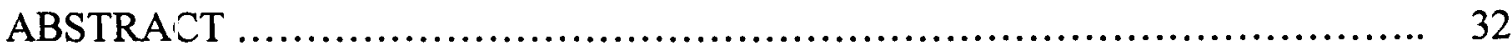

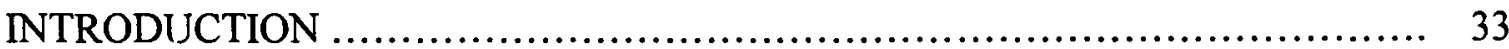

EXPERIMENT 1

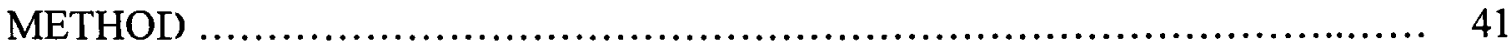

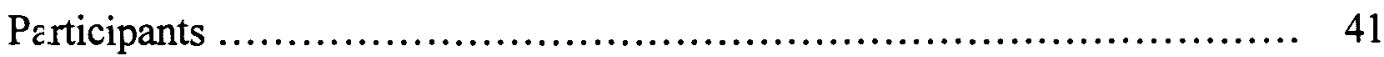

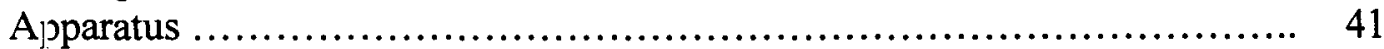

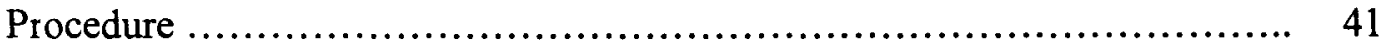

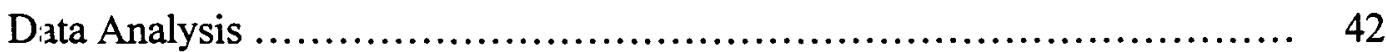

Dependent Variables ................................................... 43

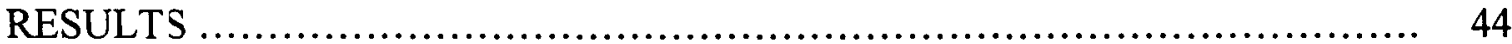

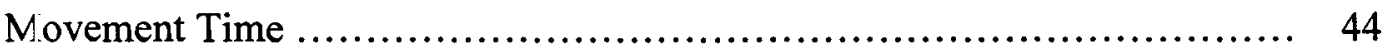

Constant Error and Variable Error ....................................... 44

Kinematic Data: Spatial Variability ................................... 45

Kinematic Data: Difference Scores ..................................... 46

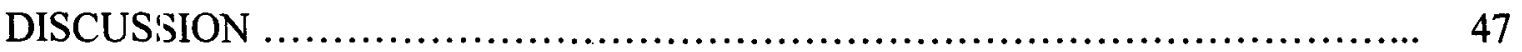

\section{EXPERIMENT 2}

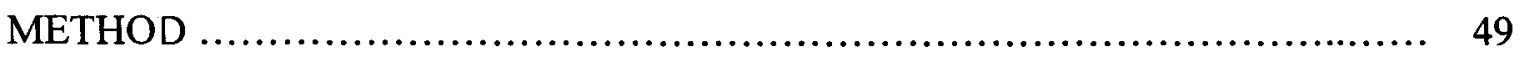

Participants ........................................................... 49

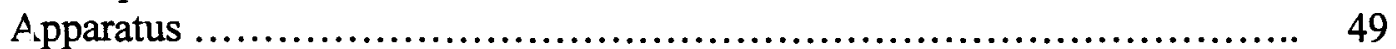

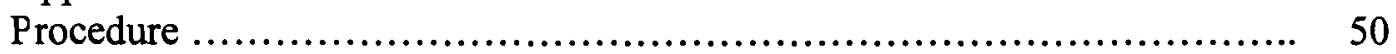

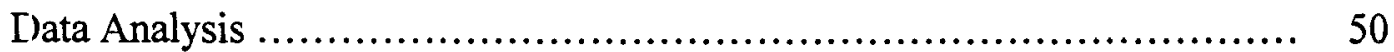

I)ependent Variables .................................................. 51

RESUL $] S$ S ................................................................. 51

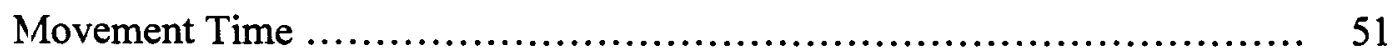

Constant Error and Variable Error ....................................... 52

Kinematic Data: Spatial Variability ................................... 53

Kinematic Data: Difference Scores .................................... . 53

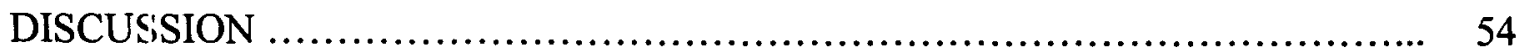


EXPERIMENT 3

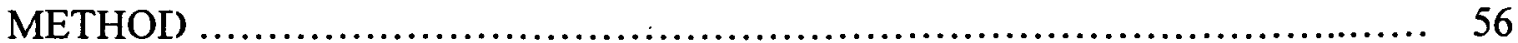

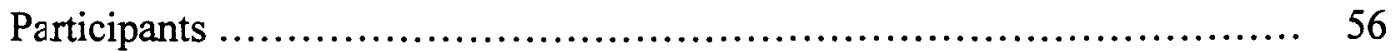

Apparatus .............................................................. 56

Procedure ................................................................ 56

Data Analysis .......................................................... 57

Dependent Variables ............................................... 57

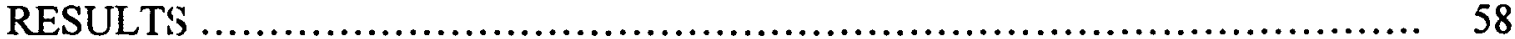

Movement Time ...................................................... 58

Constant Error and Variable Error ................................... 58

Kinematic Data: Spatial Variability ........................................ 59

Kinematic Data: Difference Scores ................................... 59

DISCUSSION ............................................................ 61

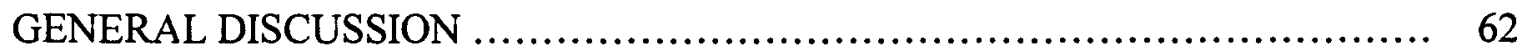

Trvo Visual Systems Hypothesis ..................................... 63

Planning-Control Hypothesis .......................................... 64

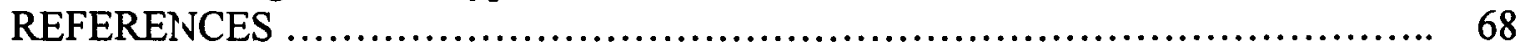

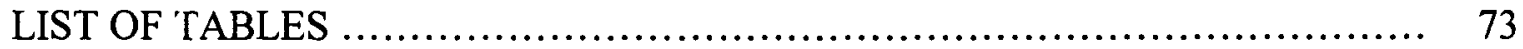

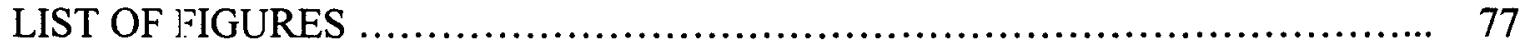




\section{GENERAL INTRODUCTION}

Vision supplies us with a wealth of information about our surroundings, allowing us to perceive the world and move efficiently. We use this information not only to make cognitive judgments about the size, shape, and use of objects, but also to guide our actions directed at these objects. A large body of behavioural evidence has suggested that the neural processes underlying perception are functionally and anatomically distinct from those underlying the visual control of actions.

\section{Two Vistal Systems}

Over the years a substantial body of research has accumulated investigating the possible existence of two potentially distinct visual systems: vision for perception and vision for action. In a set of experiments performed 35 years ago, Schneider (1969) observed that hamsters with lesions in their primary visual cortex were incapable of pattern discrimination but were still able to orient towards objects. Lesions in the subcortical system (superior colliculus), however, produced opposite effects. Schneider postulated a functional dichotomy: a cortical system that identified visual stimuli and a subcortical system that located the stimuli in space. This influential work provided the foundation for research that continues today.

Similarly, Ungerleider and Mishkin (1982) identified two distinct streams of processing in the cerebral cortex of the macaque monkey brain: a ventral stream projecting from the occipital cortex to the inferior temporal cortex and a dorsal stream projecting to the posterior parietal cortex. They observed that monkeys with lesions in the inferior temporal cortex were unable to discriminate between objects of different 
shapes. Le:sions in the posterior parietal cortex, however, left the monkeys incapable of performing landmark discrimination tasks. Ungerleider and Mishkin (1982) concluded that the ventral stream plays a role in object vision, enabling the monkey to identify an object ("what" pathway) whereas the dorsal stream is involved with object location, enabling the monkey to localize the object in space ("where" pathway).

More recently, Milner and Goodale (1995) have re-interpreted this dichotomy, proposing that the neural processing for perception and the control of action occur in the ventral and dorsal streams respectively. Unlike Ungerleider and Mishkin (1982), however, this view places less emphasis on incoming visual information distinctions (i.e., "what" versus "where") than it does on the differences in the transformations that the streams perform upon that information. Analysis of visual information in the ventral stream constructs long-term perceptual representations of the world and the objects within it, allowing us to recognize and identify objects we have previously encountered. To form such representations, the perceptual system uses object-based information (i.e., an allocentric frame of reference) to compute an object's size, shape, and location relative to other objects in the scene. This is not the case for the dorsal stream, which uses visual information to support actions directed at these objects. This system analyzes the absolute size of the object, as well as its orientation and position with respect to the observer (i.e., an egocentric frame of reference). It is important that the visuomotor system computes an object's real size, in order for us to reach and pick it up efficiently. Because the position of the target object in the action space of an observer is rarely constant. computations must be carried out every time an action occurs, and must be 
updated during the execution of that action. There is no sense in storing the egocentric coordinates and their resulting motor programs for more than a brief amount of time. As a result, transformations within the dorsal stream are carried out on-line, in real-time, producing skillfully controlled actions.

\section{Perception Versus Action}

Many insights into the dissociation of the two visual streams have come from studies on patients who have damage to one of the two systems. For example, Milner, Dijkerman, Pisella, McIntosh, Tilikete, Vighetto, and Rossetti (2001) observed an optic ataxic patient I.G., who suffered damage to her posterior parietal cortex. She demonstrated intact ventral stream function with impaired dorsal stream function. I.G. was unable to appropriately scale her handgrip when directly reaching out to pick up objects of different sizes. Despite this deficit, however, she was fairly accurate when asked to manually estimate the size of the same objects using her forefinger and thumb. I.G.'s preserved perceptual abilities accompanied with visuomotor difficulties suggest a dissociation between the ventral and dorsal streams of visual processing.

This pattern contrasts strikingly with patients suffering from visual agnosia. Extensive studies have been performed on patient D.F., who suffered damage to her inferior :emporal cortex from carbon monoxide poisoning. D.F.'s ability to visually discriminate between geometric forms, copy simple line drawings, and recognize objects, letters, and digits is severely impaired. Thus, D.F.'s pattern of deficit is largely restricted to problems in form perception (i.e., ventral stream function). In contrast, D.F. exhibits preserved visuomotor abilities (i.e., dorsal stream function). In a well-documented study 
(Goodale, Milner, Jakobson, \& Carey, 1991), a slot was cut out of a vertically mounted disc, and was oriented at varying angles on different trials. D.F.'s attempts to make a perceptual report of the orientation of the slot (verbally or by manually setting a comparison slot) showed little relationship to its actual orientation. In contrast, she was able to accurately orient her hand in order to insert a card through the slot. This impaired ventral stream function with preserved dorsal stream function provides further support for a dissociation between the visual systems.

In healthy subjects, considerable research has compared judgmental responses to motor responses to the same stimuli in order to establish the existence of separate visual streams. One of the clearest examples of a dissociation comes from experiments in which the position of a target is moved unpredictably during a saccadic eye movement. In an early study, Bridgeman, Lewis, Heit, and Nagle (1979) showed that when targets were moved slightly during a saccade, participants failed to detect these displacements on a cognitive level (i.e., verbally or by button press). In contrast, pointing movements were directed to the true location of the target. In a similar study, Goodale, Pélisson, and Prablanc (1986) replicated these results. Participants were asked to move their finger from a central target to a small target that appeared in their peripheral visual field. The target eithler remained in the same position (normal trials) or was displaced to a new location cluring the first saccadic eye movement (displaced trials). Similar to Bridgeman et al. (1979), participants failed to report the change in position in the latter condition, but continued to direct their pointing movements to the new location of the target. 
Complementary dissociations in perception and action have also been observed in experiments utilizing induced motion and the Roelofs effect. Bridgeman, Kirch, and Sperling (1981) have shown that even though a fixed target surrounded by a moving frame appizars to drift in the direction opposite to that of the frame, participants persist in pointing to the veridical location of the target. In a related study, Wong and Mack (1981) reported that saccades made to visible targets were driven by their actual location. Participants were presented with a target that was centred within a frame. Following a 500 ms blank period, the target and frame reappeared, with the frame displaced a few degrees tc the left or right. While the target was reported as moving in the direction opposite to that of the frame, saccades were directed to the actual location and not the perceived location of the target. In a more recent study, Bridgeman, Perry, and Anand (1997) utilized the Roelofs effect, which causes a target position to be misperceived when it is surrounded by a frame presented asymmetrically. For example, a frame displayed to the left of: a participant's midline will make the target inside the frame appear farther to the right "han its actual position. On each trial, a target and frame were presented simultancously for 1 second and then extinguished. The participants were asked to make a cognitive judgment (i.e., keyboard press) or a pointing movement to the location of the previous.y visible target. When there was no delay between stimulus exposure and the cue to respond, the effect always influenced perception, but not pointing.

Even when a change in the position of the target object was perceived in these experiments, movements were uninfluenced by these perceptions. There is evidence that the neural processing that underlies perception takes longer than the processing that 
underlies action. Castiello, Paulignan, and Jeannerod (1991) required participants to grasp a dowel that was suddenly displaced at the onset of the movement. Participants were also asked to indicate the time they became aware of its displacement by a simple vocal utterance. On displacement trials, corrections to the trajectory occurred as little as $100 \mathrm{~ms}$ after movement initiation, whereas the vocal response occurred $420 \mathrm{~ms}$ after the onset of the movement. The authors concluded that this temporal dissociation reflects a dissociation in neural pathways: visual processing for perceptual awareness of a stimulus is distinct from, and takes longer than processing the motor acts to the same stimulus.

Perhaps the largest line of evidence supporting a double dissociation comes from studies that utilize visual illusions. According to Milner and Goodale (1995), illusions are a result of perceptual processing that occur wholly in the ventral stream, and that they do not affect actions because they are processed independently. One such illusion is the Ebbinghaus (or Titchener circles) illusion, in which two targets of equal size are each surrounded by an array of either smaller or larger circles. The target circle surrounded by the array of smaller circles is perceived to be larger than the one surrounded by the array of larger circles. In a well-known study, Aglioti, DeSouza, and Goodale (1995) utilized thin plastic discs as the centre circles, and arranged them side-by-side in a typical Ebbinghaus array on a display card. In one condition the centre discs appeared to be equal in size although they were physically different, while in the other condition the centre discs appeared to be different in size although they were physically identical. The participants were required to pick up the disc on the left if they thought the discs were the same size, and the disc on the right if they thought the discs were different. Throughout 
testing, perticipants remained sensitive to the illusion. That is, they treated discs that were physically different as perceptually the same, and discs that were physically identical as perceptually different. In contrast, the kinematic data indicated that maximum grip aperture was scaled appropriately to the true size of the disc. For example, even when the participants perceived the discs as being the same size, they had a wider grip aperture for the larger disc than they did for the smaller one. In short, grip aperture was refractory to the effect of the size-contrast illusion.

Recently, Haffenden and Goodale (1998) replicated these findings. In this experiment, however, participants were tested under open-loop conditions (i.e., no vision of the hand or target) to eliminate the possibility of on-line control of grip aperture. Furthermore, rather than a same/different choice (cf., Aglioti et al., 1995), the perceptual task required the participants to estimate the size of the centre disc by matching the distance between their thumb and index finger. A clear dissociation was observed between the calibration of grip aperture and the perceptual estimates. When reaching out and picking up the disc, participants scaled their grip to its actual size even though they did not have the opportunity to use visual feedback. In contrast, manual estimates were biased in the direction of the illusion.

In summary, there is substantial evidence that supports the suggestion that there is a functional dissociation between perception and action. Milner and Goodale (1995) attribute this dissociation to the independent transformation of incoming visual informction in the ventral and dorsal streams. The ventral stream is specialized for making cognitive judgments regarding the size, shape, and relative location of objects. 
The dorsal stream, on the other hand, is largely responsible for the control of goaldirected movement, and is a faster operating system that is often immune from the influence of cognitive executive control. While perceptions are formed using objectcentred information that takes into consideration the surrounding scene, actions use egocentric information that computes the absolute position of the target. This explains the accurcte movements despite biased perceptions in the aforementioned studies. There are certain circumstances, however, in which actions are driven by the perceived rather than the egocentric position of a target.

\section{Perception Influences Action}

Evidence that actions are not always independent of perception comes from studies where actions are driven from memory. It has been found that saccades made to the remembered locations of targets were less accurate than saccades to visible targets (Gnadt, Bracewell, \& Andersen, 1991; White, Sparks, \& Stanford, 1994). Wong and Mack (1981) demonstrated that memory-driven saccades were driven by the target's perceived position. Similar to their first experiment, participants were presented with a target within a displaced frame, giving the illusion that the target moved in the direction opposite to that of the frame. The participants were required to make a first saccade to the visible target, and then a second "look back" saccade to its remembered location, after the target and frame disappeared. Replicating the results from the first experiment, the first saccade was directed to the actual location of the target. In contrast, the second, memory-driven saccade corresponded to the perceived displacement of the target. 
Therefore, saccades directed from memory were determined by the relative location of the target within the frame rather than its absolute location in egocentric space.

Sirnilar biases have been found in aiming movements when a no-vision delay between stimulus presentation and the cue to respond has been introduced. Elliott and Madalena (1987) reported a drastic deterioration in aiming performance when participants were required to wait 2 seconds in the dark prior to movement initiation. The results suggest that information useful to the control of movement persists for a brief amount o.: time (i.e., less than 2 seconds) following visual occlusion. Bradshaw and Watt (2002) rejlicated these findings. In this experiment, a target was presented for 1 second and then extinguished. Participants were instructed to point to the remembered location of the target after a $0-, 1-, 2-$, or 4 -second no-vision delay. In addition to pointing, the participants were also asked to perceptually match the position of the target by directing a beam from a laser pointer to where they thought the target was located. These perceptual matches were unaffected by the delays, but pointing responses clearly deteriorated after only 2 seconds.

In the Bridgeman et al. (1997) study that utilized the Roelofs effect, motor responses initiated after a 4- or 8-second delay corresponded to the perceived location of the target. The absence of a dissociation between perception and action when a delay is introduced support the suggestion that the dorsal stream has a very limited short-term memory. The no-vision delay forced the participants to use information that was biased by the f:ame position to guide their movements. In other words, the memory 
requirements associated with a no-vision delay makes participants dependent on the stored representations formed in the ventral stream.

The no-vision delay also affects movement bias in reaching and grasping movements. Hu and Goodale (2000) presented participants with a target object (a threedimensional block), accompanied with a smaller or larger block. The participants were required to pick up the target block in real-time or after a 5 second delay period. In the former condition, recording of hand movements revealed that grip aperture was not affected by the size of the accompanying block. In the latter condition, however, grip aperture vas larger when the target block was paired with a smaller object than when it was paired with a larger object. A similar size-contrast effect was found when participarts were asked to manually estimate the size of the target block. The results from this experiment give support to the suggestion that visuomotor control relies on an egocentric (i.e., absolute) frame of reference while delayed grasping uses an allocentric (i.e., relative) frame of reference.

Similar results have been found in illusion studies. For example, the closed $(\longleftrightarrow)$ and open $(><)$ configurations of the Müller-Lyer illusion, where the horizontal line is perceived as being shorter or longer than a control figure (-), respectively, have biasing $\mathrm{effects}$ on action when executed after a no-vision delay. Elliott and Lee (1995) demonstrated that the magnitude of the aiming bias towards the Müller-Lyer illusion increased when there was a 2 second no-vision delay between stimulus presentation and movement initiation. That is, compared to a full vision condition, participants undershot the closed configuration and overshot the open configuration to a greater extent when the 
delay was introduced. These results were replicated in a study by Gentilucci, Chieffi, Daprati, Saetti, and Toni (1996), who also investigated pointing movements directed to the Müller-Lyer illusion in four conditions: full vision of the stimulus and the pointing hand, vision of the stimulus but not the hand, no vision of either with a 0 second delay, and no vision of either with a 5 second delay before pointing. Similar to Elliott and Lee (1995), aining movements were biased in a manner consistent with their influence on perceptual judgments. Participants undershot the closed configuration (i.e., perceived as shorter) and overshot the open configuration (i.e., perceived as longer). There was a gradual increasing effect of the illusion when pointing was executed from memory compared to the full vision condition. In terms of the two visual systems, the results suggest a greater reliance on the ventral stream as memory became more involved.

In a recent study, Westwood, Chapman, and Roy (2000) compared natural and pantomimed actions toward the closed and open configurations of Müller-Lyer stimuli. In this experiment, a three-dimensional bar was placed over the shaft of the illusion. In the natural condition, participants viewed the array for 2 seconds, after which an auditory cue signaled them to initiate their response. Full vision was available for the duration of the response. In the pantomime condition, participants viewed the target for 2 seconds, after which vision was occluded for 3 seconds. After this delay, an auditory cue sounded and full vision was restored for the duration of the response. In each condition, the participaats were asked to perform an action task and a perceptual task. The former required participants to grasp the bar, while the latter required them to separate their thumb and index finger to the perceived width of the bar. Results revealed a dissociation 
between action and perception in the natural but not pantomime condition. That is, kinematic data in the natural condition indicated that perception was biased by the illusion bul: action was not. In the pantomime condition, kinematic data indicated that the effect of the illusion was similar for action and perception.

It is: clear from these studies that movement accuracy is severely degraded when movements are executed from memory. Milner and Goodale (1995) attribute this susceptibility to the ventral-dorsal dichotomy. The transformations that occur in the ventral stream encode information about a target in an allocentric frame of reference. Therefore. induced motion, the Roelofs effect, and visual illusions produce biased representations because it takes into account the surrounding context (i.e., a surrounding frame, closed/open arrowheads). Transformations in the dorsal stream, however, process the absolute features of the object in egocentric coordinates as the action is executed. Memory is relatively unimportant for the control of actions, because the relation between observer and target is constantly changing. Therefore, after a considerable delay between stimulus presentation and movement onset (i.e., more than 2 seconds), the egocentric coordinates would have decayed and, as a result, the stored representations formed in the ventral stream are used to guide the movement.

\section{Evidence Against A Dissociation}

A.lthough there is plenty of evidence that supports Milner and Goodale's (1995) two visual systems dichotomy, recent findings have contradicted this view. It has been found that saccadic eye movements are biased by the closed and open configurations of the Müller-Lyer illusion (Binsted \& Elliott, 1999a; Binsted \& Elliott, 1999b; Binsted, 
Chua, Helsen, \& Elliott, 2001). In a study by Binsted and Elliott (1999a), participants were instructed to move their fixation between the endpoints of Müller-Lyer stimuli and two control figures ( + and $\square$ ) in time with a metronome. Their results revealed that both the initial saccade and the end position of the eyes following corrective saccades were biased by the illusory endpoints in the expected direction. In a subsequent study, Binsted and Elliott (1999b) examined saccadic and manual movements toward closed and open Müller-Lyer endpoints, and a control figure $(X)$. Their results indicated that eye movements were biased by the illusion, but neither the primary movement of the hand nor its end position was affected. Even when the target was eliminated upon movement initiation, eye movements continued to be biased by the illusion. The illusion did, however, have an effect on the trajectory and final position of the hand when the target disappeared. Therefore, unlike hand movements, eye movements exhibited a pattern more consistent with an allocentric frame of reference (i.e., ventral stream), than an egocentric frame of reference (i.e., dorsal stream).

Pavani, Boscagli, Benvenuti, Rabuffetti, and Farnè (1999) found that perceptual estimates and grasping were similarly influenced by the Ebbinghaus illusion. Unlike the study by Aglioti et al. (1995), either a large-, small-, or neutral-circles array was presented one at a time. In the perceptual task, participants were asked to choose from a series of reference discs the one that was identical in size to the centre disc of the array. Participants were also required to reach and grasp the centre disc. The results of this study indicated that both perceptual judgments and grasping were biased by the illusion. That is, participants underestimated the size of the centre disc in the large-circles array 
(i.e., smaller grip aperture) and overestimated the size in the small-circles array (i.e., larger grip aperture) relative to the neutral condition.

Recently, it has been found that the Müller-Lyer illusion has biasing effects on grasping (Franz, Fahle, Bülthoff, \& Gegenfurtner, 2001) and aiming movements (Meegan, (Glazebrook, Dhillon, Tremblay, Welsh, \& Elliott, 2003). For example, Meegan et al. (2003) had participants view a $30 \mathrm{~cm}$ tails-in (i.e., closed), tails-out (i.e., open) or taill-less (i.e., control) Müller-Lyer stimulus, after which a random dot mask was presented for $10 \mathrm{~ms}$ or $3000 \mathrm{~ms}$. In the perceptual task, participants indicated whether a compariscn line was shorter, longer, or the same length as the shaft of the Müller-Lyer stimulus. In the motor task, participants moved a stylus to where they thought the opposite $\epsilon$ nd of the figure was located. Participants performed the motor task with and without vision of the moving limb. The results indicated the participants' perceptions were relizbly biased by the illusion. That is, they underestimated the length of the tails-in figure and overestimated the length of the tails-out figure. In contrast to Milner and Goodale's (1995) expectations, the pattern of such illusion-induced bias in the motor protocol was similar between the $10 \mathrm{~ms}$ and $3000 \mathrm{~ms}$ delay conditions. That is, participants undershot the tails-in configuration and overshot the tails-out configuration relative to the control regardless of the length of the delay period. The two visual systems hypothesis predicts an influence of the illusion in the $3000 \mathrm{~ms}$ delay condition because movements depend on ventral stream function, but not for movements in the 10 ms delay condition, which are under the control of the dorsal stream. 
In summary, there is considerable evidence that calls into question Milner and Goodale's (1995) two visual systems hypothesis. Taken together, the results suggest that actions governed by the dorsal stream are susceptible to visual illusions. These studies indicate that the transformation of visual information for perception and action are not entirely independent from each other. It seems as though the computations made for perceptual judgments can dominate those made for the on-line control of actions, producing, biased movements. Given these findings, alternative models have been developed to explain the effect of illusions on action. Recently, the planning-control model arçues that the dissociation is more accurately described as one between planning and on-line control (Glover, 2002) as opposed to ventral and dorsal stream function. Specifically, this model suggests that visual illusions only affect the planning of actions but not o:-2line control (Glover, 2002; Glover \& Dixon, 2001a; Glover \& Dixon, 2001b; Glover \&: Dixon, 2001c; Glover \& Dixon, 2002).

\section{The Planning-Control Hypothesis}

In several grasping studies, Glover and Dixon (2001a, 2001b, 2001c) observed an illusion-nduced bias only during the early portions of the movement. A threedimensional bar was placed at varying angles on a background grating that was oriented either $11^{\circ}$ clockwise $\left(+10^{\circ}\right.$ condition), or $10^{\circ}$ counterclockwise $\left(-10^{\circ}\right.$ condition) from the participants' midline. This induced an orientation illusion: when the grating was clockwise from vertical, the bar was perceived to be rotated slightly more counterclockwise, and vise versa. When the participants grasped the bar, the illusion had a larger effect on the orientation of the hand in the early portion of the reach than the later 
portion. That is, by the time the reach was completed, hand posture reflected the actual orientation of the bar, rather than its perceived orientation. This occurred whether or not the participants had vision of the target and their hand during the reach (Glover \& Dixon, 2001b).

Similar to Milner and Goodale (1995), Glover and Dixon's model assumes two separate visual representations. Rather than perception and action, however, the dichotomy is one between planning and control. Glover (in press) argues that the planning phase occurs prior to movement initiation, with the goal of selecting an appropriate motor program and kinematic parameters. Planning involves an analysis of the spatial characteristics of the target (i.e., its size, shape, orientation, and location relative to the observer), the non-spatial characteristics of the target (i.e., its weight and fragility), and its surrounding context. As a consequence, errors occur in the plan when the context induces a visual illusion, and will be apparent in the early portions of the movement. To ensure spatial accuracy upon movement termination, the control system corrects for such errors on-line. Unlike the planning system, on-line control only takes into account the spatial characteristics of the target since these likely change or are erroneously planned. Therefore, the control system is only able to correct actions that are related tc characteristics such as grip aperture (size), hand posture (orientation), and movement amplitude (relative location). With reference to Glover and Dixon's grasping studies, the planning system used a context-dependent visual representation that took into consideration the background grating, resulting in a biased hand orientation early on in the reach. During the execution of the movement, however, the control system 
disregarded the surrounding array by using a context-independent visual representation to produce an accurate hand posture by the time the movement was completed.

The control system is able to compute and update a visual representation quite rapidly by using a combination of visual feedback, proprioceptive feedback, and efference copy (i.e., a "blueprint" of the motor plan sent to the control system). This allows for fast on-line adjustments to be made, but also means that the control system has a limited memory. As a result, the visual representation formed by the control system decays when a 2 second no-vision delay between stimulus presentation and movement onset is irtroduced (cf., Elliott \& Madalena, 1987). Movements that are initiated after a delay of rnore than 2 seconds remain uncorrected and are executed as planned. In contrast, the more time the visual and proprioceptive feedback loops have to operate, the more time on-line control can exert its effect on action. For example, short-duration movements are carried out as planned, while long-duration movements are under the influence of the control system.

Support for this view has come mostly from grasping studies utilizing the Ebbinghaus illusion (Glover \& Dixon, 2002) and the Müller-Lyer illusion (Westwood, Heath, \&: Roy, 2000; Westwood, McEachern, \& Roy, 2001). Glover and Dixon (2002) suggested that the small effect on grasping reported in previous illusion studies (Aglioti et al., 1995; Haffenden \& Goodale, 1998) was because only peak grip aperture, an event that occurs late in the movement, was measured. It is possible that the illusion affected the planning of the movements but these effects were corrected on-line. Therefore, they analyzed the grip aperture throughout the course of a reaching movement toward the 
centre disc of the Ebbinghaus illusion. This provided an indication of how the grasps were planned (i.e., early stage of the movement) from its effects on how the grasps were controlled (i.e., later stage of the movement). Their results showed that regardless of whether or not vision was available, the illusion had a large effect early in the reach and decreased as the movement progressed to completion. Westwood et al. (2000) analyzed peak apercure velocity (an early event) and peak grip aperture (a late event) of grasping movements made toward a three-dimensional bar placed within the Müller-Lyer illusion. Their results indicated that the former was largely influenced by the illusion while the latter reflected the true size of the bar. Taken together, these results are supportive of the planning-control hypothesis because the kinematic events that reflected planning were biased by illusions, while the events that reflected control were immune to their effects. However, several studies have not supported this view. For example, Danckert, Sharif, Haffenden, Schiff, and Goodale (2002) reanalyzed kinematic data from an earlier experiment (Haffenden, Schiff, \& Goodale, 2001) to determine whether the Ebbinghaus illusion had a biasing effect throughout the course of a movement. When they compared grip aperture to the small- and large-circles array at four different time points $(25 \%, 50 \%$, $75 \%$, and $100 \%$ of the total time to reach peak aperture), there was no illusion-induced bias even at the earliest stages of the grasp. Meegan et al. (2003) measured the bias at early (i.e., peak velocity) and late kinematic markers (i.e., peak deceleration and movement endpoint) to determine the effect of the Müller-Lyer illusion on aiming. Their results indicated that although the participants were engaging in on-line control late in the movement (Khan, Lawrence, Fourkas, Franks, Elliott, \& Pembroke, 2003), it was not 
enough to overcome the bias induced by the Müller-Lyer illusion. That is, movements towards the tails-in and tails-out configurations moved a lesser and greater distance compared to the control condition, respectively, even at the latter stage of the movement.

From the amount of conflicting evidence, it is evident that the debate over the visual processes that underlie perception and action is far from over. Although the planning-control hypothesis attempts to explain the influence of illusions on action that Milner and Goodale's (1995) two visual systems model cannot explain, there is also significant research that is inconsistent with the planning-control dichotomy. The purpose of the following experiments is to further investigate the two hypotheses discussed here, in an attempt to settle the debate. 


\section{REFERENCES}

Aglioti, S., DeSouza, J.F.X., \& Goodale, M.A. (1995). Size-contrast illusions deceive the eye but not the hand. Current Biology, 5, 679-685.

Binsted, G., Chua, R., Helsen, W., \& Elliott, D. (2001). Eye-hand coordination in goaldirected aiming. Human Movement Science, 20, 563-585.

Binsted, (J., \& Elliott, D. (1999a). The Müller-Lyer illusion as a perturbation to the saccadic system. Human Movement Science, 18, 103-117.

Binsted, (G., \& Elliott, D. (1999b). Ocular perturbations and retinal/extraretinal information: the coordination of saccadic and manual movements. Experimental Brain Research, 127, 193-206.

Bradshaw, M.F., \& Watt, S.J. (2002). A dissociation of perception and action in normal human observers: the effect of temporal-delay. Neuropsychologia, 40, 17661778.

Bridgeman, B., Lewis, S., Heit, G., \& Nagle, M. (1979). Relation between cognitive and motor-oriented systems of visual position perception. Journal of Experimental Psychology: Human Perception and Performance, 5, 692-700.

Bridgeman, B., Kirch, M., \& Sperling, A. (1981). Segregation of cognitive and motor aspects of visual function using induced motion. Perception and Psychophysics, $29,336-342$.

Bridgeman, B., Perry, S., \& Anand, S. (1997). Interaction of cognitive and sensorimotor maps of visual maps. Perception and Psychophysics, 59, 456-469.

Castiello, U., Paulignan, Y., \& Jeannerod, M. (1991). Temporal dissociation of motor 
responses and subjective awareness. Brain, 114, 2639-2655.

Danckert, J.A., Sharif, N., Haffenden, A.M., Schiff, K.C., \& Goodale, M.A. (2002). A temporal analysis of grasping in the Ebbinghaus illusion: planning versus online control. Experimental Brain Research, 144, 275-280.

Elliott, D., \& Madalena, J. (1987). The influence of premovement visual information on manual aiming. The Quarterly Journal of Experimental Psychology, 39A, 541559.

Franz, V.H., Fahle, M., Bülthoff, H.H., \& Gegenfurtner, K.R. (2001). Effects of Visual Illısions on Grasping. Journal of Experimental Psychology: Human Perception and Performance, 27, 1124-1144.

Gentilucci, M., Chieffi, S., Daprati, E., Saetti, M.C., \& Toni, I. (1996). Visual illusion and action. Neuropsychologia, 34, 369-376.

Glover, S. (2002). Visual illusions affect planning but not control. Trends in Cognitive Sciences, 6, 288-292.

Glover, S. (in press). Separate visual representations in the planning and control of action. Behavioral and Brain Sciences.

Glover, S., \& Dixon, P. (2001a). Dynamic illusion effects in a reaching task: evidence for separate representations in the planning and control of reaching. Journal of Experimental Psychology: Human Perception and Performance, 27, 560-572.

Glover, S., \& Dixon, P. (2001b). The role of vision in the on-line correction of illusion e:fects on action. Canadian Journal of Experimental Psychology, 55, 96-103.

Glover S., \& Dixon, P. (2001c). Motor adaptation to an optical illusion. Experimental 
Brain Research, 137, 254-258.

Glover, S., \& Dixon, P. (2002). Dynamic effects of the Ebbinghaus illusion in grasping: supjort for a planning/control model of action. Perception and Psychophysics, $64,266-278$.

Gnadt, J.W., Bracewell, R.M., \& Andersen, R.A. (1991). Sensorimotor transformation during eye movements to remembered visual targets. Vision Research, 31, 693715.

Goodale, M.A., Milner, A.D., Jakobson, L.S., \& Carey, D.P. (1991). A neurological dissociation between perceiving objects and grasping them. Nature, 349, 154156.

Goodale, M.A., Pélisson, D., \& Prablanc, C. (1986). Large adjustments in visually guided reaching do not depend on vision of the hand or perception of target displacement. Nature, 320, 748-750.

Haffenden, A.M., \& Goodale, M.A. (1998). The effect of pictorial illusion on prehension and perception. Journal of Cognitive Neuroscience, 10, 122-136.

Haffenden, A.M., Schiff, K.C., \& Goodale, M.A. (2001). The disṣociation between perception and action in the Ebbinghaus illusion: nonillusory effects of pictorial cules on grasp. Current Biology, 11, 177-181.

Hu, Y., \& Goodale, M.A. (2000). Grasping after a delay shifts size-scaling from absolute to relative metrics. Journal of Cognitive Neuroscience, 12, 856-868.

Khan, M.A., Lawrence, G., Fourkas, A., Franks, I.M., Elliott, D., \& Pembroke, S. (2J03). Online versus offline processing of visual feedback in the control of 
movement amplitude. Acta Psychologica, 113, 83-97.

Meegan, D.V., Glazebrook, C.M., Dhillon, V.P., Tremblay, L., Welsh, T.N., \& Elliott, D. (2003). The Müller-Lyer illusion affects the planning and control of manual air.ing movements. Experimental Brain Research, 155, 37-47.

Milner, A..J., Dijkerman, H.C., Pisella, L., McIntosh, R.D., Tilikete, C., Vighetto, A., \& Rossetti, Y. (2001). Grasping the past: delay can improve visuomotor performance. Current Biology, 11, 1896-1901.

Milner A.D., \& Goodale M.A. (1995). The visual brain in action. Oxford University Press, Oxford.

Pavani, F., Boscagli, I., Benvenuti, F., Rabuffetti, M., \& Farnè, A. (1999). Are pe:ception and action affected differently by the Titchener circles illusion? Experimental Brain Research, 127, 95-101.

Schneider, G.E. (1969). Two visual systems: brain mechanisms for localization and discrimination are dissociated by tectal and cortical lesions. Science, 163, 8959012.

Ungerleicler, L.G., \& Mishkin, M. (1982). Two cortical visual systems. In D.J. Ingle, M.A. Goodale, \& R.J.W. Mansfield (Eds.), Analysis of visual behavior (pp. 5495i36). MIT Press, Cambridge, MA.

Westwocid, D.A., Chapman, C.D., \& Roy, E.A. (2000). Pantomimed actions may be controlled by the ventral visual stream. Experimental Brain Research, 130, 545548.

Westwond, D.A., Heath, M., \& Roy, E.A. (2000). The effect of a pictorial illusion on 
clo:sed-loop and open-loop prehension. Experimental Brain Research, 134, 456463

Westwood, D.A., McEachern, T., \& Roy, E.A. (2001). Delayed grasping of a MüllerLyer figure. Experimental Brain Research, 141, 166-173.

White, J.M., Sparks, D.L., \& Stanford, T.R. (1994). Saccades to remembered target locations: an analysis of systematic and variable errors. Vision Research, 34, 7992.

Wong, E., \& Mack, A. (1981). Saccadic programming and perceived location. Acta Ps,chologica, 48, 123-131. 
The Effect of the Müller-Lyer Illusion on the

Planning and Control of Manual Aiming Movements

Jocelyn E. Mendoza

Department of Kinesiology

McMaster University 


\begin{abstract}
The following experiments were conducted to investigate the predictions of two visual systems hypothesis (Milner \& Goodale, 1995) and the planning-control model (Glover, 2(102) on manual aiming movements to the vertices of tails-in, no tails, and tailsout Müller-Lyer stimuli. Experiment 1 required the participants to initiate their movements after a $0 \mathrm{~ms}$ or $5000 \mathrm{~ms}$ no-vision delay. In Experiment 2, the participants executed their movements with full vision to Müller-Lyer stimuli that either remained the same or changed to a different configuration upon movement initiation. Experiment 3 was identical to Experiment 2 except that the amount of visual feedback for on-line control was constrained with liquid crystal goggles to $200 \mathrm{~ms}, 400 \mathrm{~ms}$, or $600 \mathrm{~ms}$. Taken together, the results are problematic for both hypotheses. Participants were susceptible to the illusion even when movements were initiated after a $0 \mathrm{~ms}$ delay period. In addition, the magnitude of the aiming bias increased as the movement unfolded (Experiment 1). Furthermore, even though participants were engaging in on-line control, the illusion continuec to exert its effects on aiming during the latter stages of the movement (Experiment 2). Interestingly, this effect was observed although participants had ample time (i.e., $600 \mathrm{~ms}$ ) to use visual feedback in order to modify their movements (Experiment 3).
\end{abstract}




\section{INTRODUCTION}

It has long been suggested that two functionally distinct streams underlie visual processing. From their work with macaque monkeys, Ungerleider and Mishkin (1982) identified a ventral and dorsal stream projecting from the primary visual cortex to the inferior temporal and posterior parietal cortex, respectively. They reported that monkeys with lesions to the inferior temporal cortex were unable to discriminate between objects, while lesions in the posterior parietal cortex led to deficits in land discrimination tasks. These findings lead Ungerleider and Mishkin (1982) to conclude that the ventral stream is responsible for identifying objects ("what" pathway) while the dorsal stream is responsible for locating objects in space ("where" pathway).

Milner and Goodale (1995) have proposed an alternative point of view, suggesting that the ventral stream underlies perception while the dorsal stream underlies the control of action. Unlike Ungerleider and Mishkin (1982) this view places less emphasis on the differences between incoming visual information (i.e., object qualities versus object location) than it does on the transformations performed upon that information (Goodale \& Milner, 1992). The ventral stream analyzes visual information to form long-term representations of the world and the objects within it, permitting us to recognize and identify objects we have encountered in the past. To do so, the ventral stream uses an allocentric frame of reference (i.e., object-based information) that analyzes the size, shape, and location of an object relative to other objects in the scene. The dorsal stream, on the other hand, uses an egocentric frame of reference (i.e., viewerbased information) that analyzes the absolute size of the object as well as its orientation 
and position in relation to the observer. For example, to pick up an object, especially one that has never been encountered before, it is not enough to know whether it is smaller or larger than accompanying objects. The object's real size must be calculated independent of its surroundings for us to reach out and pick it up efficiently. Furthermore, because the position of the object with reference to the observer is constantly changing as the movement unfolds, the dorsal stream must continually update the position of the object during the: execution of that action. As a consequence, the dorsal stream has a limited memory since it stores the egocentric coordinates for only a brief period of time.

Over the years there has been considerable evidence to support a dissociation between the two visual systems. One of the clearest examples comes from studies of visual agnosic patient D.F., who suffered damage to her inferior temporal cortex from carbon monoxide poisoning. In a study by Goodale, Milner, Jakobson, and Carey (1991), a slot was; cut out of a vertically mounted disc, and was oriented at varying angles on different trials. Even though D.F. was unable to match the orientation of the slot (verbally or by manually setting a comparison slot), she accurately oriented her hand in order to insert a card through the slot. Despite her deficits in perceptual estimates (ventral stream function) her visuomotor ability (dorsal stream function) remained intact.

Another line of evidence that supports a dissociation comes from studies utilizing visual illusions. According to Milner and Goodale (1995), illusions are a result of perceptual processing that occurs entirely in the ventral stream. Thus, they are not expected to affect actions. In a well-cited study, Aglioti, DeSouza, and Goodale (1995) used the Ebbinghaus (or Titchener circles) illusion, in which two circles of equal size 
appear to be smaller or larger if they are surrounded by an array of large or small circles, respectively. Throughout testing, the participants' perceptions remained sensitive to the illusion. In contrast, when participants reached out and grasped the target disc, their maximum grip aperture was scaled appropriately to the true size of the disc. In a similar study by Flaffenden and Goodale (1998), these findings were replicated under open-loop conditions (i.e., no vision of the target or the hand). Taken together, these results suggest that actiors are immune to the effect of visual illusions whether or not vision is available during the movement. There are certain circumstances, however, in which actions are driven by our perceptions rather than the true qualities of the object.

Evidence that actions are not always independent of perception comes from studies where actions are driven from memory. It has been found that saccadic eye movements (Gnadt, Bracewell, \& Andersen, 1991; White, Sparks, \& Stanford, 1994; Wong \& Mack, 1981), aiming movements (Bradshaw \& Watt, 2002; Elliott \& Lee, 1995; Elliott \& Madalena, 1987; Gentilucci, Chieffi, Daprati, Saetti, \& Toni, 1996), and grasping rnovements (Hu \& Goodale, 2000; Westwood, Chapman, \& Roy, 2000) are less accurate vrhen they are initiated after a no-vision delay between stimulus presentation and the cue to respond than when vision is eliminated upon movement initiation. Elliott and Madalena (1987) reported some deterioration in movement accuracy and consistency when vision was eliminated upon movement initiation, and a drastic deterioration in aiming performance when a delay of as little as 2 seconds was introduced. Gentilucci et al. (1996) utilized the closed $(\longleftrightarrow)$ and open $(\succ<)$ configurations of the Müller-Lyer illusion, where the horizontal line is perceived to be shorter or longer, respectively. They 
observed that the magnitude of the aiming bias increased when the participants executed their movements after a 5 second delay. Compared to a full vision condition, participants undershot the closed configuration and overshot the open configuration to a greater extent when the clelay was introduced. Milner and Goodale (1995) would attribute these results to the stored representations formed in the ventral stream, which are susceptible to visual illusions. After an appreciable delay, the egocentric coordinates would have decayed and, as a result, participants must instead depend on the biased representations to guide their movements.

Despite the large amount of evidence supporting Milner and Goodale's (1995) ventral-dorsal dichotomy, several current findings are not consistent with this view (Binsted \& Elliott, 1999; Franz, Fahle, Bülthoff, \& Gegenfurtner, 2001; Pavani, Boscagli, Benvenuti, Rabuffetti, \& Farnè, 1999). In a recent study by Meegan, Glazebrook, Dhillon, 'Tremblay, Welsh, and Elliott (2003), participants were asked to point to the vertex of previously visible Müller-Lyer stimuli after a $10 \mathrm{~ms}$ or $3000 \mathrm{~ms}$ delay. It was reported that participants undershot the closed configuration and overshot the open configuration relative to a control figure, regardless of delay condition. The two visual systems hypothesis predicts an influence of the illusion in the $3000 \mathrm{~ms}$ delay condition because movements depend on ventral stream function, but not for the $10 \mathrm{~ms}$ delay condition, which should still be under the control of the dorsal stream. Perhaps as Franz et al. (2001) have suggested, a single representation is used for perceptual decisionmaking and the guidance of actions. 
From the amount of conflicting evidence associated with the two visual systems hypothesis, an alternate dual process model has been proposed in order to explain the effect of illusions on action. Recently, the planning-control hypothesis suggests that the dichotomy does not lie between perception and action but between planning and on-line control (Cilover, 2002). Specifically, (Glover, 2002) argues that visual illusions only affect the planning of actions while on-line control remains immune to their effects (Glover \&: Dixon, 2001a; Glover \& Dixon, 2001b; Glover \& Dixon, 2001c; Glover \& Dixon, 2002).

In several grasping studies, Glover and Dixon (2001a, 2001b, 2001c) observed an illusion-induced bias only during the early portions of the movement. They utilized an orientation illusion, where a vertical bar placed on slanted background grating appeared to be rotated in the opposite direction. They reported that the orientation of the hand early in the grasp was biased in the direction of the illusion, but final hand posture reflected the actual orientation of the bar.

Glover (2002) suggested that movements are planned and initiated through a context-dependent visual representation that incorporates a target's surrounding array. Therefore, errors in the plan occur when the context induces an illusion. While the movement is being executed, however, the control system corrects for these errors through a visual representation that ignores the surrounding context. To ensure spatial accuracy, the control system updates the representation quite rapidly by using a combination of visual feedback, proprioceptive feedback, and efference copy. This allows :for fast on-line adjustments to be made, but also means that the control system has 
a limited memory. As a result, any movements initiated after a 2 second delay will remain uncorrected because the visual representation would have decayed during this interval. In contrast, the more time the visual and proprioceptive feedback loops have to operate, the more the control system can exert its effects on action. Many studies have supported this suggestion (Westwood, Heath, \& Roy, 2000; Westwood, McEachern, \& Roy, 200:) while others have not (Danckert, Sharif, Haffenden, Schiff, \& Goodale, 2002; Meegan et al., 2003). For example, Meegan et al. (2003) measured the bias at early (i.e., peak velocity) and late (i.e., peak deceleration, movement end) kinematic markers to determine the effect of the Müller-Lyer illusion on aiming. They reported that even though participants were engaging in on-line control (Khan, Lawrence, Fourkas, Franks, Elliott, \& Pembroke, 2003), it was not enough to overcome the illusion-induced bias. That is, novements towards the closed and open configurations moved a lesser and greater distance compared to the control figure, respectively, even at the latter portion of the movement.

The two visual systems hypothesis and the planning-control model were further investigated in the following experiments. Participants were asked to rapidly point to the right ver.ex of tails-in $(\longleftrightarrow)$, no tails $(\longrightarrow)$, and tails-out $(><$ ) Müller-Lyer stimuli under a variety of conditions. In Experiment 1, participants initiated their movements after a $0 \mathrm{~ms}$ or $5000 \mathrm{~ms}$ no-vision delay between stimulus presentation and movement onset. The two visual systems hypothesis predicts that movements in the former condition would still be regulated by the dorsal stream and will not be influenced by the illusion, while movements in the latter condition should show an illusion-induced bias 
because they are guided by perceptual representations in the ventral stream. To test the planning-control hypothesis, we examined the presence of on-line control as well as illusion-induced bias at early (planning) and late (control) kinematic markers. Studies performed by Khan et al. (2003) reported that most on-line regulation of the limb occurs between peak deceleration and the end of the movement. Given this finding, the planning-control hypothesis predicts that the illusion will have a larger impact at kinematic markers associated with a planning (e.g., peak velocity) than at those associated with control (e.g., peak deceleration and movement end). It also predicts that the illusicn will not influence kinematic markers associated with control for the $0 \mathrm{~ms}$ delay but will for the $5000 \mathrm{~ms}$ delay, since the context-independent representation will have decalyed during this time period. In Experiment 2, we examined the illusioninduced bias of movements made to changing Müller-Lyer stimuli. One configuration of the illusion was presented during planning and it either remained the same or changed to a different configuration upon movement initiation. According to Glover and Dixon (2001a), any bias would reflect the configuration viewed during planning, and would be larger in early portions of the movement trajectory than later portions of the movement regardless of the configuration seen during execution. Experiment 3 was similar to Experiment 2, except that we constrained the amount of visual feedback to $200 \mathrm{~ms}, 400$ ms, or $6100 \mathrm{~ms}$ during movement execution. Studies performed by Zelaznik, Hawkins, and Kisselburgh (1983), found that visual feedback is beneficial for aiming movements less than $120 \mathrm{~ms}$ in duration. Given this, the planning-control model predicts that on-line 
control would be able to correct for errors in planning, especially when ample feedback (i.e., $600 \mathrm{~ms}$ ) is available. 


\title{
EXPERIMENT 1
}

\author{
Method
}

\section{Participants}

Ten undergraduate and graduate students (age range 20-32 years) from the McMaster University community participated in this study. Participants were righthanded, had normal or corrected-to-normal vision, and were naive to the purpose of the study.

Apparatus

E.Prime software was used to generate the Müller-Lyer stimuli. A projector mounted $100 \mathrm{~cm}$ above the surface projected the stimuli onto a piece of black bristol board, which was placed on top of a wooden board. Participants held a stylus in their right hand, and placed it on a switch embedded in the wooden board. An infrared emitting diode (IRED) was attached to the tip of the stylus. An auditory tone triggered an Optotrak $\cdot 3020$ system (Northern Digital Inc.) to begin recording the position of the IRED for $1500 \mathrm{~ms}$ at a rate of $200 \mathrm{~Hz}$. Liquid crystal goggles were used to occlude vision of the limb during the delay period, and for an additional $1500 \mathrm{~ms}$ from the time the auditory tone sounded.

\section{Procedure}

The participants began each trial by depressing the switch with the stylus. Participents were presented a $25 \mathrm{~cm}$ or $35 \mathrm{~cm}$ Müller-Lyer stimulus for $1000 \mathrm{~ms}$; the location of the switch coincided with the left vertex of the illusion. Vision was then occluded for $0 \mathrm{~ms}$ or $5000 \mathrm{~ms}$ with the liquid crystal goggles. Following this delay, an 
auditory signal sounded, signaling the participants to point to where they thought the right apex of the Müller-Lyer stimulus was located. The auditory signal triggered the Optotrak to record for $1500 \mathrm{~ms}$. Participants were instructed to keep the stylus in the final position until the goggles became transparent again (i.e., the Optotrak was finished recording). Factorial combinations of Müller-Lyer configuration (tails-in, no tails, tailsout), Length $(25 \mathrm{~cm}, 35 \mathrm{~cm})$, and Delay $(0 \mathrm{~ms}, 5000 \mathrm{~ms})$ were presented 10 times in random order for a total of 120 trials. The tails of the Müller-Lyer stimuli were oriented $45^{\circ}$ from the horizontal, and were $5 \mathrm{~cm}$ in length for $25 \mathrm{~cm}$ stimuli or $7 \mathrm{~cm}$ in length for $35 \mathrm{~cm}$ stimuli.

Data Anclysis

Displacement data from the Optotrak were filtered using a dual-pass Butterworth filter with a cut-off frequency of $10 \mathrm{~Hz}$. Movement in the primary direction (Y-axis) was analyzed using custom software (Chua \& Elliott, 1993). The difference between the finish and start locations was calculated to determine the total displacement in the $\mathrm{Y}$-axis. Displacement and velocity were differentiated to generate velocity and acceleration profiles, respectively. The start and end of the movement were identified as the frame-at which velocity rose above or fell below $30 \mathrm{~mm} / \mathrm{s}$, respectively, and remained there for 70 ms. The custom software identified a number of kinematic markers in the $\mathrm{Y}$-axis velocity and acceleration profiles, and the spatial location at which these kinematic events cccurred. This allowed for the examination of spatial variability and the amplitude bias throughout the trajectory. 
Tc calculate constant and variable error, we subtracted the movement endpoint location f:om the known target location. Constant error, the mean algebraic error in the Y-axis, determined whether the participant undershot (i.e., negative error) or overshot (i.e., positive error) the target amplitude. Variable error, the standard deviation of these spatial endpoints in the $\mathrm{Y}$-axis, reflected within-participant consistency of aiming movements. To determine whether the participants were engaging in on-line control late in the movement, spatial variability at peak acceleration (PA), peak velocity (PV), peak deceleration (PD), and movement end (variable error) was calculated.

\section{Dependent Variables}

Movement time, constant error, and variable error were analyzed using a 3 Configu:ation (tails-in, no tails, tails-out) by 2 Length $(25 \mathrm{~cm}, 35 \mathrm{~cm})$ by 2 Delay $(0 \mathrm{~ms}$, $5000 \mathrm{~ms}$ ) repeated measures analysis of variance (ANOVA). The spatial variability measures at each kinematic marker were analyzed using a 4 Kinematic Marker (PA, PV, PD, end) by 3 Configuration (tails-in, no tails, tails-out) by 2 Length $(25 \mathrm{~cm}, 35 \mathrm{~cm})$ by 2 Delay $(0 \mathrm{~ms}, 5000 \mathrm{~ms})$ repeated measures ANOVA. To examine the aiming bias throughout the trajectory, the amplitude achieved at PA, PV, PD, and movement endpoint was determined. Using the mean of the no tails stimuli as the standard in each condition, difference scores were calculated for aiming movements made to the tails-in and tails-out stimuli. A positive difference score meant the participant moved a greater distance relative: to the control situation, while a negative difference score meant the participant moved a lesser distance relative to the no tails stimuli. These scores were analyzed using a 4 Kinematic Marker (PA, PV, PD, end) by 2 Configuration (tails-in, tails-out) by 2 
Length $(25 \mathrm{~cm}, 35 \mathrm{~cm})$ by 2 Delay $(0 \mathrm{~ms}, 5000 \mathrm{~ms})$ repeated measures ANOVA.

Tukey's HSD $(p<.05)$ test was used to post hoc significant effects involving more than two means.

\section{Results}

\section{Movement Time}

This analysis revealed main effects for Configuration, $F(2,18)=5.05, p<.05$, Length, $F^{\prime}(1,9)=22.60, p<.001$, and Delay, $F(1,9)=6.89, p<.05$ (Table 1$)$.

Participants moved significantly faster when presented tails-in stimuli (421 ms) than when presiented with no tails stimuli $(430 \mathrm{~ms}$; tails-out: $423 \mathrm{~ms})$. They were also faster in completing their movements toward $25 \mathrm{~cm}$ stimuli $(406 \mathrm{~ms})$ than toward $35 \mathrm{~cm}$ stimuli (443 ms) and after a $0 \mathrm{~ms}$ delay $(408 \mathrm{~ms})$ than after a $5000 \mathrm{~ms}$ delay $(440 \mathrm{~ms})$. There was also a significant interaction of Configuration and Length, $F(2,18)=4.02, p<.05$. The difference in movement times between the two lengths was more pronounced when the participants were presented with no tails stimuli $(25 \mathrm{~cm}: 405 \mathrm{~ms} ; 35 \mathrm{~cm}: 454 \mathrm{~ms})$. Constant Error and Variable Error

The constant error analysis yielded main effects for Configuration, $F(2,18)=$ $51.80, p<.001$ and Delay, $F(1,9)=12.02, p<.01$ (see Table 1). Participants undershot the tails-in stimuli $(-36 \mathrm{~mm})$ to a greater degree than the tails-out stimuli $(-12 \mathrm{~mm})$, with the no tails configuration being intermediate $(-23 \mathrm{~mm})$. There was also a significantly greater undershooting effect when participants made aiming movements after a $5000 \mathrm{~ms}$ no-vision delay $(-31 \mathrm{~mm} ; 0 \mathrm{~ms}:-16 \mathrm{~mm})$. There was a tendency to undershoot $35 \mathrm{~cm}$ stimuli $(-28 \mathrm{~mm})$ more so than $25 \mathrm{~cm}$ stimuli $(-19 \mathrm{~mm})$, however this effect was not 
significant, $F(1,9)=3.74, p=.08$. The analysis also revealed an interaction of Configuration and Length, $F(2,18)=5.99, p<.05$. Participants undershot $35 \mathrm{~cm}$ tails-in and no tails stimuli to a greater extent compared to the same $25 \mathrm{~cm}$ configurations $(25 \mathrm{~cm}$ tails-in: $-29 \mathrm{~mm} ; 35 \mathrm{~cm}$ tails-in: $-42 \mathrm{~mm} ; 25 \mathrm{~cm}$ no tails: $-18 \mathrm{~mm} ; 35 \mathrm{~cm}$ no tails: -28 $\mathrm{mm})$. However, this difference disappeared when presented with tails-out stimuli $(25 \mathrm{~cm}$ tails-out: $-11 \mathrm{~mm} ; 35 \mathrm{~cm}$ tails-out: $-12 \mathrm{~mm}$ ). There was also a significant three-way interaction of Configuration, Length, and Delay, $F(2,18)=6.70, p<.01$ (Figure 1). After a 0 ms delay, there was a significant difference between $25 \mathrm{~cm}$ and $35 \mathrm{~cm}$ stimuli only when pointing to the tails-in configuration. However, after a $5000 \mathrm{~ms}$ no-vision delay, a significant difference between the two lengths was seen with movements to tailsin as well. as no tails stimuli. Interestingly, the analysis failed to yield a Configuration by Delay interaction, $F(2,18)=1.32, p=.29$. The participants exhibited a similar illusioninduced bias in both delay conditions, indicating that there was no dissociation between memory-based (ventral stream) and on-line (dorsal stream) processing. This is contrary to the two visual systems hypothesis (Milner \& Goodale, 1995).

The variable error analysis only revealed a main effect for Delay, $F(1,9)=30.14$, $p<.001$ (see Table 1). Movements executed after the $0 \mathrm{~ms}$ delay $(21 \mathrm{~mm}$ ) were significantly more consistent than those made after the $5000 \mathrm{~ms}$ delay $(28 \mathrm{~mm})$. Kinemaitic Data: Spatial Variability

Analysis of the spatial variability measures at PA, PV, PD, and movement endpoint confirmed the occurrence of on-line control late in the movement trajectory (Khan et al., 2003). This analysis revealed a main effect of Kinematic Marker, $F(3,27)=$ 
$18.66, p<.001$. There was a drastic increase in spatial variability between PV and PD, and a pronounced decrease between PD and the end of the movement. The analysis also revealed interactions of Kinematic Marker and Length, $F(3,27)=4.18, p<.05$ and Kinematic Marker and Delay, $F(3,27)=9.83, p<.001$. There was a significant difference in spatial variability between movements towards $25 \mathrm{~cm}$ and $35 \mathrm{~cm}$ stimuli only at PA. (Figure 2). Also, the variability of movements made after a 0 ms compared to after a 50(10 ms delay was reliably different at PD and movement end (Figure 3).

\section{Kinematic Data: Difference Scores}

One of the main features of the planning-control hypothesis is the ability of the control system to correct for illusion-induced errors made during planning. As the spatial variability data indicates, on-line control occurred late in the movement trajectory. However, the constant error data suggests that this was not enough to overcome the illusion-induced bias. The difference score analysis revealed a main effect for Configuration, $F(1,9)=69.36, p<.001$. Relative to the no tails configuration, participants undershot the target amplitude for tails-in stimuli and overshot the amplitude for tails-out stimuli (Figure 4). The analysis also yielded interactions of Kinematic Marker and Configuration, $F(3,27)=41.65, p<.001$ as well as Configuration and Delay, $F(1,9)=11.33, p<.01$. The magnitude of the illusion-induced bias increased at each kinematic marker, showing a progressively undershooting effect for the tails-in figure and overshovting effect for the tails-out figure. The bias at PD and movement endpoint was reliably greater than that at PA and PV for tails-in stimuli, while the bias at movement endpoint was significantly greater than that at PA for tails-out stimuli (Figure 5). Also, 
undershoo:ing tails-in stimuli and overshooting tails-out stimuli was more pronounced after a $5000 \mathrm{~ms}$ no-vision delay (tails-in: $-8 \mathrm{~mm}$; tails-out: $8 \mathrm{~mm}$ ) than after no delay (tails-in: $-7 \mathrm{~mm}$; tails-out: $4 \mathrm{~mm}$ ).

\section{Discussion}

The purpose of this study was to examine the effect of a no-vision delay on aiming movements towards Müller-Lyer stimuli. Based on the two visual systems hypothesi:s, it was hypothesized that there would be no illusion-induced bias in the $0 \mathrm{~ms}$ delay while movements initiated after a $5000 \mathrm{~ms}$ delay would exhibit an illusion-induced bias. Also, the magnitude of the aiming bias was expected to decrease as the movement unfolded, reflecting the occurrence of on-line control (Glover \& Dixon, 2001a).

The results, however, did not support these predictions. First, a strong illusioninduced tias was observed in both delay conditions, contrary to the two visual systems hypothes:s. Second, kinematic analyses revealed that the aiming bias actually increased as the movement progressed to completion. This occurred despite the fact that on-line control was occurring late in the movement trajectory. The latter findings are problematic for the planning-control model, which suggests that control processes should reduce the effect of an illusion during the execution of a movement. The findings from the current study replicate results reported by Meegan et al. (2003), who found a similar pattern of illusion-induced bias when aiming movements were executed after $10 \mathrm{~ms}$ and $3000 \mathrm{~ms}$ delays, as well as an increasing bias as the movement unfolded.

A main difference between the current study and the study performed by Meegan et al. (2003) was the use of liquid crystal goggles to occlude vision during the delay 
period. In the latter experiment, a random dot mask was presented between stimulus presentation and the cue to respond. The participants could have benefited from the availability of such context by choosing a location on the board to direct their movements. This strategy might diminish any difference between memory-based aiming and on-line control. In the current study, we eliminated all environmental cues via the liquid crysial goggles to ensure that movements in the $5000 \mathrm{~ms}$ delay condition were truly exectited from memory. Interestingly, our results paralleled those of Meegan et al. (2003), inclicating that the effect of the illusion persists, regardless of whether or not context is available during this period.

In addition to Milner and Goodale's hypothesis (1995), the current experiment presented evidence against the model put forward by Glover (2002). Experiment 2 examined the planning-control hypothesis in greater detail. By separately manipulating the configurations seen during planning and control, we can determine the effect of the illusion dıring each of these stages. 


\section{EXPERIMENT 2}

The purpose of this study is to investigate the effect of changing Müller-Lyer stimuli on manual aiming. Previous studies have introduced a perturbation upon movement: initiation to examine adjustments in the movement trajectory (Heath, Hodges, Chua, \& Elliott, 1998; Paulignan, MacKenzie, Marteniuk, \& Jeannerod, 1991; Pélisson, Prablanc, Goodale, \& Jeannerod, 1986; Proteau \& Masson, 1997). In the current study, the initial Müller-Lyer configuration changed to a different one upon movement initiation on the majority of trials. Glover (2002) predicts that regardless of the configuration viewed during the execution of the movement, on-line control can correct for the errors made during the planning phase. Any illusion-induced bias will reflect the "planning configuration", and will be evident in the early portions of the movement. As the movement progresses to completion (i.e., while on-line control is operating), the magnitude of the bias will decrease.

Method

\section{Participants}

Twelve graduate and undergraduate students (age range 20 to 32 years) from the McMaster University community participated in this study. Participants were righthanded, had normal or corrected-to-normal vision, and were naïve to the purpose of this study.

Apparatus

The apparatus was similar to the one used in Experiment 1. 


\section{Procedure}

Participants began each trial by depressing the switch with the stylus. They were presented a $25 \mathrm{~cm}$ or $35 \mathrm{~cm}$ Müller-Lyer stimulus for $1000 \mathrm{~ms}$ ("preparation configuration"); the location of the switch coincided with the left vertex of the illusion. An auditory tone then signaled the participants to move to the opposite vertex of the illusion as quickly and accurately as possible. The auditory tone triggered the Optotrak to record for $1500 \mathrm{~ms}$. Upon releasing the switch (i.e., movement initiation), the illusion either changed configuration or remained the same ("execution configuration"). To be certain that participants were making rapid movements, vision was occluded $350 \mathrm{~ms}$ (for $25 \mathrm{~cm}$ stimuli) or $450 \mathrm{~ms}$ (for $35 \mathrm{~cm}$ stimuli) after movement initiation with liquid crystal goggles. These values were chosen based on the average movement times obtained from Experiment 1. Participants were instructed to remain in the final position until the goggles became transparent again (i.e., Optotrak finished recording). Factorial combinations of Length $(25 \mathrm{~cm}, 35 \mathrm{~cm})$, Preparation Configuration (tails-in, no tails, tails-out), and Execution Configuration (tails-in, no tails, tails-out) were presented 10 times in random order for a total of 180 trials. The tails on the Müller-Lyer stimuli were oriented $45^{\circ}$ from the horizontal, and were $5 \mathrm{~cm}$ in length for $25 \mathrm{~cm}$ stimuli and $7 \mathrm{~cm}$ in length for $35 \mathrm{~cm}$ stimuli.

Data Analysis

Data collected from the Optotrak were analyzed using the same criteria and techniques as in Experiment 1. 


\section{Dependent Variables}

Movement time, constant error, and variable error were analyzed using a 2 Length ( $25 \mathrm{~cm}, 35 \mathrm{~cm}$ ) by 3 Preparation Configuration (tails-in, no tails, tails-out) by 3 Execution Configuration (tails-in, no tails, tails-out) repeated measures ANOVA. Spatial variability measures were analyzed using a 4 Kinematic Marker (PA, PV, PD, movement end) by 2 Length $(25 \mathrm{~cm}, 35 \mathrm{~cm}$ ) by 3 Preparation Configuration (tails-in, no tails, tails-out) by 3 Execution Configuration (tails-in, no tails, tails-out) repeated measures ANOVA. The amplitude achieved at PA, PV, PD, and movement endpoint was determined to examine the aiming bias as the movement unfolded. The mean for the control ccndition (prepared and executed with no tails) was used as the standard, and difference: scores were calculated for aiming movements in the remaining 8 conditions. Difference scores were analyzed using a 4 Kinematic Marker (PA, PV, PD, movement end) by 2 Length $(25 \mathrm{~cm}, 35 \mathrm{~cm})$ by 8 Condition (prepared tails-in and executed tails-in, tails-in and no tails, tails-in and tails-out, no tails and tails-in, no tails and tails-out, tailsout and tails-in, tails-out and no tails, tails-out and tails-out) repeated measures ANOVA. Tukey's HSD $(p<.05)$ test and separate planned comparisons were used to post hoc significant effects.

\section{Results}

\section{Movement Time}

This analysis revealed a main effect of Length, $F(1,11)=39.89, p<.001$ (Table 2 ) and a Length by Preparation Configuration interaction, $F(2,22)=3.79, p<.05$. Participants were significantly faster in completing their movements when aiming 
towards $2.5 \mathrm{~cm}$ stimuli $(395 \mathrm{~ms})$ than towards $35 \mathrm{~cm}$ stimuli $(438 \mathrm{~ms})$. Also, the differences in movement time towards the two lengths were more pronounced when the participants viewed a tails-in configuration during planning $(25 \mathrm{~cm}: 392 \mathrm{~ms} ; 35 \mathrm{~cm}: 443$ $\mathrm{ms})$.

\section{Constant Error and Variable Error}

The constant error analysis yielded main effects for Length, $F(1,11)=36.39, p<$ .001 , Prejaration Configuration, $F(2,22)=14.98, p<.001$, and Execution Configuration, $F(2,22):=53.82, p<.001$ (see Table 2). Participants significantly undershot $35 \mathrm{~cm}$ stimuli $(-4.35 \mathrm{~mm})$ to a greater extent than $25 \mathrm{~cm}$ stimuli $(-0.38 \mathrm{~mm})$. Movements that were planned with the tails-in and no tails configurations undershot the target amplitude the most, and differed significantly from movements prepared with the tails-out configuration. With respect to execution, participants undershot the tails-in configuration the most, and differed significantly from the tails-out configuration, which was undershot the least. The constant error of movements executed toward no tails stimuli were intermediate and were reliably different from those executed to tails-in and tails-out stimuli (Figure 6).

The variable error analysis yielded a main effect of Execution Configuration, $F(2$, $22)=4.44, p<.05$ (see Table 2). Movements executed with the tails-in $(6.16 \mathrm{~mm})$ and no tails configurations $(6.23 \mathrm{~mm})$ were significantly more consistent than those toward the tails:-out configuration $(7.24 \mathrm{~mm})$. 


\section{Kinematic Data: Spatial Variability}

This analysis revealed a main effect for Kinematic Marker, $F(3,33)=26.74, p<$ .001 , confirming the occurrence of on-line control late in the movement. Spatial variability increased drastically between $\mathrm{PV}$ and $\mathrm{PD}$, then decreased sharply between $\mathrm{PD}$ and movement end (Figure 7). The variability at movement end differed significantly from all cther kinematic markers.

\section{Kinemati: Data: Difference Scores}

The difference score analysis revealed main effects of Kinematic Marker, $F(3,33)$ $=10.27, p<.001$, and Condition, $F(7,77)=15.01, p<.001$. An interaction of Kinematic Marker and Condition, $F(21,231)=4.44, p<.001$ was also found. Relative to the control condition, participants undershot at PA and overshot at the remaining kinematic markers. The bias at PD was reliably greater than that at PA and PV. The bias at movement end, however, only differed significantly from PA. Planned comparisons revealed that movements prepared with tails-in were shorter than those prepared with tails-out. $F(1,11)=50.58, p<.001$, and movements executed with tails-in were also shorter than those executed with tails-out, $F(1,11)=13.29, p<.01$. Movements prepared with no tails and executed with tails-in differed reliably from those prepared with no tails and executed with tails-out, $F(1,11)=14.28, p<.01$ (Figure 8). For the two-way interaction, planned comparisons were carried out at each kinematic marker. Participants moved a lesser distance when they prepared their movements with tails-in stimuli than when having prepared with tails-out stimuli at PV, $F(1,11)=51.21, p<.001, \mathrm{PD}, F(1$, $11)=24.46, p<.001$, and at the termination of the movement, $F(1,11)=16.64, p<.01$ 
(Figure 9). Movements executed with the tails-in configuration were also shorter than those executed with the tails-out configuration at $\mathrm{PD}, F(1,11)=9.90, p<.01$, and at movement end, $F(1,11)=72.57, p<.001$ (Figure 10). Also, movements prepared with no tails and executed with tails-in moved a lesser distance than those prepared with no tails and executed with tails-out at PA, $F(1,11)=6.72, p<.05, \mathrm{PD}, F(1,11)=5.11, p<$ .05 , and at the end of the movement, $F(1,11)=24.17, p<.001$ (see Figure 10).

\section{Discussion}

The purpose of the second experiment was to investigate the influence of changing: Müller-Lyer stimuli on manual aiming movements. Based on the planningcontrol hypothesis (Glover, 2002), it was predicted that any illusion-induced bias would reflect the configuration viewed during planning, and should be evident only during the early portion of the movement. During execution, on-line control would exert its effects and correct for planning errors, resulting in an unbiased hand position by the end of the movement. Contrary to expectations, the configuration of the illusion seen during executicn had a biasing effect on late kinematic markers. That is, on-line control of longduration movements (approximately $450 \mathrm{~ms}$ ) was susceptible to the illusion. This result is problematic for the planning-control hypothesis, which suggests that control is immune to the surrounding context.

In the grasping studies performed by Glover and Dixon, average movement times were relatively long (i.e., greater than $500 \mathrm{~ms}$ ). This, perhaps, gave participants sufficient time to overcome the effects of the illusion. Although Zelaznik et al. (1983) found that visual feedback can be processed for movement times less than $120 \mathrm{ms,} \mathrm{it}$ 
could be argued that the constraints of $350 \mathrm{~ms}$ and $450 \mathrm{~ms}$ of visual feedback in the current experiment was not enough to eliminate all of the illusion-induced bias. The next experiment addressed this issue. 


\section{EXPERIMENT 3}

The purpose of this experiment is to investigate the effect of changing MüllerLyer stimuli on aiming movements. The experimental set-up is similar to Experiment 2, except that the amount of visual feedback was constrained to $200 \mathrm{~ms}, 400 \mathrm{~ms}$, or $600 \mathrm{~ms}$ during execution. According to Zelaznik et al. (1983) visual feedback can be processed for movement times as little as $120 \mathrm{~ms}$. The planning-control model (Glover, 2002) predicts that movements will be carried out as planned for the former two conditions, but that on-line control will correct for errors in planning when ample feedback (i.e., $600 \mathrm{~ms}$ ) is availatile.

\section{Method}

\section{Participcints}

Twelve graduate and undergraduate students (age range 18 to 32 years) from the McMaster University community participated in this study. Participants were righthanded, had normal or corrected-to-normal vision, and were naïve to the purpose of the study.

Apparatus

The apparatus was similar to Experiment 1.

\section{Procedure}

Farticipants began each trial by depressing the switch with the stylus. They were presented a $30 \mathrm{~cm}$ Müller-Lyer stimulus for $1000 \mathrm{~ms}$ ("preparation configuration"); the location of the switch coincided with the left vertex of the illusion. Upon hearing an auditory tone, participants were instructed to rapidly move the stylus to the right vertex of 
the figure. The tone triggered the Optotrak to record for $1500 \mathrm{~ms}$. Upon releasing the switch (i.e., movement initiation), the stimulus either changed configuration or remained the same ("execution configuration"). The amount of visual feedback was manipulated using the liquid crystal goggles. Vision was occluded $200 \mathrm{~ms}, 400 \mathrm{~ms}$, or $600 \mathrm{~ms}$ after movement initiation. Participants were instructed to remain in the final position until the goggles became transparent again (i.e., Optotrak finished recording). The amount of visual feedback was blocked (Elliott \& Allard, 1985; Zelaznik et al., 1983) and counterbalanced across participants, while factorial combinations of Preparation Configuration (tails-in, no tails, tails-out) and Execution Configuration (tails-in, no tails, tails-out) were randomly presented 10 times in each block for a total of 270 trials. The tails of the Müller-Lyer stimuli were oriented $45^{\circ}$ from the horizontal, and were $6 \mathrm{~cm}$ in length.

Data Analysis

Data collected from the Optotrak were analyzed using the same criteria and techniques used in Experiment 1.

Dependent Variables

Movement time, constant error, and variable error were analyzed using a 3 Visual Feedback ( $200 \mathrm{~ms}, 400 \mathrm{~ms}, 600 \mathrm{~ms}$ ) by 3 Preparation Configuration (tails-in, no tails, tails-out) by 3 Execution Configuration (tails-in, no tails, tails-out) repeated measures ANOVA.. Spatial variability and difference scores were analyzed using a 4 Kinematic Marker (PA, PV, PD, end) by 3 Visual Feedback ( $200 \mathrm{~ms}, 400 \mathrm{~ms}, 600 \mathrm{~ms})$ by 3 Preparation Configuration (tails-in, no tails, tails-out) by 3 Execution Configuration 
(tails-in, no tails, tails-out) repeated measures ANOVA. Difference scores were analyzed using a 4 Kinematic Marker (PA, PV, PD, movement end) by 3 Visual Feedback (200 ms, $400 \mathrm{~ms}, 600 \mathrm{~ms}$ ) by 8 Condition (prepared tails-in and executed tails-in, tails-in and no tails, tails-in and tails-out, no tails and tails-in, no tails and tails-out, tails-out and tailsin, tails-out and no tails, tails-out and tails-out) repeated measures ANOVA. Tukey's HSD $(p<.05)$ test and separate planned comparisons were used to post hoc significant effects.

\section{Results}

Movemen: Time

This analysis revealed a main effect for Visual Feedback, $F(2,22)=22.19, p<$ .001 (Table 3). Movement times became significantly longer as the amount of visual feedback increased, indicating that the participants utilized feedback when it was available (200 ms: $407 \mathrm{~ms} ; 400 \mathrm{~ms}: 458 \mathrm{~ms} ; 600 \mathrm{~ms}: 491 \mathrm{~ms})$.

\section{Constant Error and Variable Error}

The analysis for constant error yielded main effects for Preparation Configuration, $F(2,22)=8.13, p<.01$, and Execution Configuration, $F(2,22)=53.55, p<.001$ (see Table 3). Collapsed over visual feedback condition, participants undershot the target the most when having prepared their movements with the tails-in configuration, and the least when they were prepared with the tails-out configuration. The bias when planning with the no tails figure was intermediate. During execution, participants significantly undershct the tails-in configuration to a greater degree compared to the no tails and tailsout configurations (Figure 11). 
The variable error analysis yielded a main effect for Visual Feedback, $F(2,22)=$ $21.86, p<.001$ (see Table 3). Movements made in the $400 \mathrm{~ms}(5.22 \mathrm{~mm})$ and $600 \mathrm{~ms}$ $(5.07 \mathrm{~mm})$ visual feedback conditions were reliably more consistent than those made with $200 \mathrm{~ms}$ of visual feedback $(7.14 \mathrm{~mm})$.

\section{Kinematic Data: Spatial Variability}

This analysis yielded a main effect of Kinematic Marker, $F(3,33)=27.34, p<$ .001 . The:e was a significant increase in variability between $P V$ and $P D$, and a significant decrease between PD and movement end, indicating that on-line regulation occurred. The variability at movement end reliably differed from all other kinematic markers (Figure 12).

\section{Kinematic Data: Difference Scores}

The difference score analysis revealed main effects for Kinematic Marker, $F(3$, $33)=6.74, p<.01$, and Condition, $F(7,77)=4.61, p<.001$, as well as an interaction of Kinematic Marker and Condition, $F(21,231)=1.81, p<.05$. Participants overshot relative to the control condition at PA and undershot the remaining kinematic markers. The bias at PD differed reliably from the bias at PA, which overshot relative to the control. Flanned comparisons determined that movements prepared with the tails-in configuration were significantly shorter than those prepared with the tails-out configuration, $F(1,11)=8.32, p<.05$, and that movements executed toward tails-in and tails-out stimuli differed significantly during execution, $F(1,11)=13.84, p<.01$. Movements prepared with no tails and executed with tails-in were shorter than those prepared with no tails and executed with tails-out, $F(1,11)=6.05, p<.01$ (Figure 13). 
For the two-way interaction, planned comparisons were conducted and revealed that participants moved a lesser distance when they prepared their movements with tails-in compared to those prepared with tails-out at $\mathrm{PV}, F(1,11)=10.23, p<.01, \mathrm{PD}, F(1,11)=$ $6.13, p<.05$, and movement end, $F(1,11)=15.51, p<.01$ (Figure 14). However, movements executed with tails-in were significantly shorter than those executed with tails-out cnly at movement end, $F(1,11)=65.91, p<.001$ (Figure 15). To determine whether tiee illusion-induced bias disappeared when participants had ample visual feedback, planned comparisons were performed in each feedback condition. When participarts have $200 \mathrm{~ms}$ of feedback, movements prepared with tails-in were shorter than those prepared with tails-out at PV, $F(1,11)=9.49, p<.05, \mathrm{PD}, F(1,11)=11.12$, $p<.01$, and movement end, $F(1,11)=15.66, p<.001$ (Figure 16). This bias was also observed at movement end with $400 \mathrm{~ms}$ of visual feedback, $F(1,11)=10.25, p<.01$ (Figure 17) and at PV, $F(1,11)=11.71, p<.01$, and movement end, $F(1,11)=6.13, p<$ .05 with $500 \mathrm{~ms}$ of visual feedback (Figure 18). Participants moved a significantly lesser distance when executing movements with tails-in than when executing with tails-out at movement end in all visual feedback conditions $[200 \mathrm{~ms}: F(1,11)=13.44, p<.01$ (Figure 19); $400 \mathrm{~ms}: F(1,11)=31.36, p<.001$ (Figure 20); $600 \mathrm{~ms}: F(1,11)=22.16$, $p<.001$ (Figure 21)]. This result is problematic for the planning-control hypothesis. While participants engaged in on-line control to reduce variability, they were unable to correct for any illusion-induced biases although ample feedback was available. This cannot be attributed to fast movement times since participants used feedback when it was 
available (see Movement Time results). Therefore, it seems that the control system does not correct for illusion-induced biases.

\section{Discussion}

The purpose of this experiment was to investigate the effect of constrained visual feedback on aiming movements to changing Müller-Lyer stimuli. Based on the planning-zontrol hypothesis, it was hypothesized that movements with $200 \mathrm{~ms}$ of feedback would be carried out as planned, since on-line control would not have sufficient time to operate. This is in stark contrast to what was expected with $600 \mathrm{~ms}$ of feedback, in that this was enough time to accurately achieve the target. From Experiment 2, it was expected that movements would show some illusion-induced bias when executed with $400 \mathrm{~ms}$ of feedback.

Contrary to the planning-control hypothesis, movements continued to be biased by the illusion even when ample feedback was available. This cannot be attributed to a strategy in which participants were preparing for the "worst-case scenario" (200 ms condition) since feedback conditions were blocked (i.e., participants knew in advance the amount of feedback they will be receiving). Since visual feedback can be processed extremely rapidly (Zelaznik et al., 1983), the $600 \mathrm{~ms}$ condition was more than enough time to reduce the effects of the illusion. 


\section{GENERAL DISCUSSION}

The three experiments reported here were designed to examine the effect of the Müller-Lyər illusion on manual aiming movements: If the ventral and dorsal streams underlie memory-based action and on-line control, respectively, then movements executed $c$ fter a long delay should exhibit a greater illusion-induced bias relative to movements initiated following brief delay. These latter movements should not be influencec by the illusion (Milner \& Goodale, 1995). On the other hand, if the dichotomy is one between planning and on-line control then the magnitude of the bias should reflect the configuration of the illusion during the planning phase. This bias should be evident in the early portion of the movement and decrease as the movement unfolds, especially when ample visual feedback is available to overcome errors in planning (Glover, in press). The configuration of the illusion viewed during execution should have no effect on late kinematic markers (i.e., the control stage).

Oגr results contrast previous work on visual illusions (Gentilucci et al., 1996; Glover \& Dixon, 2001a, 2001b, 2001c, 2002; Westwood et al., 2000; Westwood et al., 2001). It was demonstrated here that: (1) movements executed after brief $(0 \mathrm{~ms})$ and long (50c0 ms) delays exhibited an illusion-induced bias (Experiment 1); (2) the magnitude of the bias increased as the movement unfolded even though participants were engaging in on-line control, as evidenced by a reduction in spatial variability between peak deceleration and the end of the movement (Experiment 1); (3) the configuration of the illusion presented during the control phase had a biasing effect on late kinematic markers (Experiments 2 and 3); and (4) on-line control continued to be biased in the 
direction of the execution configuration even when participants had ample time (i.e., 600 ms) to process visual feedback (Experiment 3). Taken together, these results are inconsistent with the predictions of the two visual systems hypothesis (Milner \& Goodale, 1995) and the planning-control hypothesis (Glover, 2002).

Two Visual Systems Hypothesis

Based on Milner and Goodale's (1995) suggestion, visuomotor control depends on the dorsal stream, which maintains an accurate representation of the environment. The ventral stream, on the other hand, is responsible for perceptual processing, and computes target characteristics (i.e., size, shape, position) relative to other objects in the scene. As evidenced by several studies, perception and action are differentially influence $\mathrm{d}$ by visual illusions, indicating that these two processes occur independently from each other (Aglioti et al., 1995; Haffenden \& Goodale, 1998). Given that the position of the target with respect to the observer is constantly changing during the execution of an action, the dorsal stream stores the target's egocentric coordinates for only a brief amount of time. Because these coordinates decay quite rapidly, the visuomotor and perceptual systems interact when movements are executed from memory (Bridgernan et al., 1997; Hu \& Goodale, 2000; Wong \& Mack, 1981). In other words, movem€nts executed after a substantial no-vision delay (i.e., 2 seconds, Elliott \& Madalena, 1987) between stimulus presentation and movement onset rely solely on perceptual memory.

Iit has been suggested, however, that visuomotor memory decays in less than 2 seconds. In a study by Westwood et al. (2000) participants grasped a bar that was 
embedded within the Müller-Lyer illusion under an open-loop condition, where liquid crystal gogggles eliminated vision of the hand and target upon the cue to respond.

Therefore, absolute object characteristics were retained in visuomotor memory for only a brief amount of time (a duration ranging from $0 \mathrm{~ms}$ to $450 \mathrm{~ms}$ prior to movement initiation, Jakobson \& Goodale, 1991). The results of this study revealed that the illusion had a biasing effect on movements in the expected direction. Given this, Westwood et al. (2000) coxcluded that visuomotor memory is significantly less than 2 seconds, and perhaps even less than $450 \mathrm{~ms}$. The authors also assume that the rate at which the visual representation in dorsal stream decays is determined by the shortest time at which the illusion biases action. Based on this assumption, the findings from the current study (0 ms delay condition in Experiment 1) suggest that the visual representation decays instantaneously. Rather than an index of visuomotor decay, perhaps the effect of the illusion after a brief delay gives us an idea about the speed at which perceptual processes provides information to the visuomotor system.

Planning-Control Hypothesis

A. possible reason why our results are not supportive of the planning-control hypothesis can be attributed to the methodological differences between the current study and those of Glover and Dixon (2001a, 2001b, 2001c, 2002). It is argued here that the methodology of the present set of experiments is more robust than that of Glover and Dixon ir examining the effects of illusions on planning and on-line control. One major difference is the illusion used in the current study. Compared to other visual illusions the Müller-J_yer illusion seems to be more effective in influencing a variety of actions, such 
as saccad c eye movements, aiming, and grasping (Binsted \& Elliott, 1999a; Binsted \& Elliott, 1999b; Elliott \& Lee, 1995; Franz et al., 2001; Gentilucci et al., 1996; Meegan et al., 2003; Westwood et al., 2000; Westwood et al., 2001). Perhaps the most apparent difference is the manipulation of the illusion configuration during the planning and control stages in the current study (Experiment 2 and 3). This allowed us to directly determine: (1) the degree to which the illusion had an influence on planning; and (2) the effect, or absence of an effect, of the execution configuration on the movement.

One of the main features of the planning-control hypothesis is that planning uses a context-clependent visual representation to generate a movement plan, while on-line control operates using a representation that is independent of the surrounding context. Results from the current set of experiments, however, suggest otherwise. A common finding was that late kinematic markers (control phase) showed no reduction in illusioninduced bias, even though participants were engaging in on-line control late in the movement. This continued to be the case even when there was sufficient time to process visual feedback (Experiment 3). Although it can be argued that the delay condition and no vision on the limb prevented the correction of on-line control, the planning-control hypothesis clearly states that such corrections can occur under these conditions (Glover, 2002).

There is reason to question the logic that on-line control ignores the context surrounding a target. Consider the example of reaching for a rose within a thick, thorny shrub. According to the planning-control hypothesis, the planning system will determine a path that will avoid contacting obstacles (thorns) along the way to the target (rose). 
Once this movement is initiated, the control system corrects for errors related to the spatial characteristics of the target. What happens if a gust of wind moves the target in an unanticips.ted way? It would make sense that on-line control, while correcting the movement, will take into account the visual context, in order to avoid the surrounding thorns. The planning-control hypothesis, however, posits that the control system will ignore the visual context (thorns) because the ultimate success of a movement depends on how accu:ate it is when the target is acquired (Glover, in press). Following this, the control system will adjust the movement in order to decrease the spatial error between target and limb, regardless of whether the limb comes into contact with the thorny surround. Based on the results reported here, it appears that the control system does in fact take nto consideration the visual context when correcting for movements on-line.

Given that the two visual systems hypothesis and the planning-control model cannot acequately explain our results, it is reasonable to call into question separate visual representation models (Franz et al., 2001). These models propose that the visual representation underlying perception is separate from the representation underlying action. Furthermore, such models argue that actions are not at all influenced by visual illusions. For example, the planning-control hypothesis proposes that illusory information is available to planning processes, but not available to on-line control. The results from the current experiment clearly contradict this suggestion. In contrast, a common. representation model (Franz et al., 2001) proposes that a single visual representation is used for perception and action guidance, and because of this, visual illusions have an effect on action. It is argued here that the present results are consistent 
with this latter model, given that the illusion had a biasing effect on aiming in the direction sonsistent with its influence on perception. On the basis of the results reported here and elsewhere (Franz et al., 2001; Meegan et al., 2003; Pavani et al., 1999), we conclude that separate representation models, such as the two visual systems hypothesis and the panning-control model, are not appropriate in explaining the effect of visual illusions on actions. 


\section{REFERENCES}

Aglioti, S., DeSouza, J.F.X., \& Goodale, M.A. (1995). Size-contrast illusions deceive the eye but not the hand. Current Biology, 5, 679-685.

Binsted, G., \& Elliott, D. (1999). The Müller-Lyer illusion as a perturbation to the sar:cadic system. Human Movement Science, 18, 103-117.

Bradshaw, M.F., \& Watt, S.J. (2002). A dissociation of perception and action in normal himan observers: the effect of temporal-delay. Neuropsychologia, 40, 17661778.

Danckert. J.A., Sharif, N., Haffenden, A.M., Schiff, K.C., \& Goodale, M.A. (2002). A temporal analysis of grasping in the Ebbinghaus illusion: planning versus online control. Experimental Brain Research, 144, 275-280.

Elliott, D., \& Allard, F. (1985). The utilization of visual feedback information during rapid pointing movements. The Quarterly Journal of Experimental Psychology, $37 A, 407-425$.

Elliott, D., \& Madalena, J. (1987). The influence of premovement visual information on manual aiming. The Quarterly Journal of Experimental Psychology, 39A, 541559.

Franz, V.H., Fahle, M., Bülthoff, H.H., \& Gegenfurtner, K.R. (2001). Effects of Visual Illusions on Grasping. Journal of Experimental Psychology: Human Perception and Performance, 27, 1124-1144.

Gentilucci, M., Chieffi, S., Daprati, E., Saetti, M.C., \& Toni, I. (1996). Visual illusion and action. Neuropsychologia, 34, 369-376. 
Glover, S. (2002). Visual illusions affect planning but not control. Trends in Cognitive Siciences, 6, 288-292.

Glover, \$. (in press). Separate visual representations in the planning and control of action. Behavioral and Brain Sciences.

Glover, S., \& Dixon, P. (2001a). Dynamic illusion effects in a reaching task: evidence for separate representations in the planning and control of reaching. Journal of Experimental Psychology: Human Perception and Performance, 27, 560-572.

Glover, S., \& Dixon, P. (2001b). The role of vision in the on-line correction of illusion effects on action. Canadian Journal of Experimental Psychology, 55, 96-103.

Glover S., \& Dixon, P. (2001c). Motor adaptation to an optical illusion. Experimental 13rain Research, 137, 254-258.

Glover, S., \& Dixon, P. (2002). Dynamic effects of the Ebbinghaus illusion in grasping: support for a planning/control model of action. Perception and Psychophysics, 64, 266-278.

Gnadt, I.W., Bracewell, R.M., \& Andersen, R.A. (1991). Sensorimotor transformation during eye movements to remembered visual targets. Vision Research, 31, 693715.

Goodale, M.A., Milner, A.D., Jakobson, L.S., \& Carey, D.P. (1991). A neurological dissociation between perceiving objects and grasping them. Nature, $349,154-$ 156.

Haffenden, A.M., \& Goodale, M.A. (1998). The effect of pictorial illusion on prehension and perception. Journal of Cognitive Neuroscience, 10, 122-136. 
Heath, M., Hodges, N.J., Chua, R., \& Elliott, D. (1998). On-line control of rapid aiming movements: Effects of target characteristics on manual aiming. Canadian Journal of Experimental Psychology, 52, 163-173.

Hu, Y., \& Goodale, M.A. (2000). Grasping after a delay shifts size-scaling from absolute to relative metrics. Journal of Cognitive Neuroscience, 12, 856-868.

Jakobson, L.S., \& Goodale, M.A. (1991). Factors affecting higher-order movement planning: a kinematic analysis of human prehension. Experimental Brain Research, 86, 199-208.

Khan, M.A., Lawrence, G., Fourkas, A., Franks, I.M., Elliott, D., \& Pembroke, S. (21003). Online versus offline processing of visual feedback in the control of movement amplitude. Acta Psychologica, 113, 83-97.

Meegan, D.V., Glazebrook, C.M., Dhillon, V.P., Tremblay, L., Welsh, T.N., \& Elliott, D. (2J03). The Müller-Lyer illusion affects the planning and control of manual aiming movements. Experimental Brain Research, 155, 37-47.

Milner A.D., \& Goodale M.A. (1995). The visual brain in action. Oxford University Press, Oxford.

Paulignan, Y., MacKenzie, C.L., Marteniuk, R.G., \& Jeannerod, M. (1991). Selective perturbations of visual input during prehension movements: I. The effects of changing object position. Experimental Brain Research, 83, 502-512.

Pavani, F., Boscagli, I., Benvenuti, F., Rabuffetti, M., \& Farnè, A. (1999). Are perception and action affected differently by the Titchener circles illusion? Experimental Brain Research, 127, 95-101. 
Pélisson, J., Prablanc, C., Goodale, M.A., \& Jeannerod, M. (1986). Visual control of reaching movements without vision of the limb: II. Evidence of fast unconscious processes correcting the trajectory of the hand to the final position of a doublestep stimulus. Experimental Brain Research, 62, 303-313.

Proteau, L., \& Masson, G. (1997). Visual perception modifies goal-directed movement control: Supporting evidence from a visual perturbation paradigm. Quarterly Jcurnal of Experimental Psychology, 50(A), 726-741.

Ungerleicler, L.G., \& Mishkin, M. (1982). Two cortical visual systems. In D.J. Ingle, M.A. Goodale, \& R.J.W. Mansfield (Eds.), Analysis of visual behovior (pp. 549 586). MIT Press, Cambridge, MA.

Westwocd, D.A., Chapman, C.D., \& Roy, E.A. (2000). Pantomimed actions may be controlled by the ventral visual stream. Experimental Brain Research, 130, 5455.48.

Westword, D.A., Heath, M., \& Roy, E.A. (2000). The effect of a pictorial illusion on closed-loop and open-loop prehension. Experimental Brain Research, 134, 456463.

Westword, D.A., McEachern, T., \& Roy, E.A. (2001). Delayed grasping of a MüllerL.yer figure. Experimental Brain Research, 141, 166-173.

White, J.M., Sparks, D.L., \& Stanford, T.R. (1994). Saccades to remembered target locations: an analysis of systematic and variable errors. Vision Research, 34, 7992.

Wong, E., \& Mack, A. (1981). Saccadic programming and perceived location. Acta 
Psychologica, 48, 123-131.

Zelaznik, J.N., Hawkins, B., \& Kisselburgh, L. (1983). Rapid visual feedback processing in single-aiming movements. Journal of Motor Behavior, 15, 217 235. 


\section{LIST OF TABLES}

Table 1. Average movement time, constant error, and variable error in

Experiment 1.

Table 2. Average movement time, constant error, and variable error in 75 Experiment 2.

Table 3. Average movement time, constant error, and variable error in

Experiment 3. 
Table 1

Ávcrage movement time (MT), constant error (CE), and variable error (VE) in Experiment 1.

\begin{tabular}{|c|c|c|c|c|c|c|c|c|c|c|c|c|}
\hline \multirow{3}{*}{$\begin{array}{l}\text { Length: } \\
\text { Delay: } \\
\text { Configuration: }\end{array}$} & \multicolumn{6}{|c|}{$25 \mathrm{~cm}$} & \multicolumn{6}{|c|}{$35 \mathrm{~cm}$} \\
\hline & \multicolumn{3}{|c|}{$0 \mathrm{~ms}$} & \multicolumn{3}{|c|}{$5000 \mathrm{~ms}$} & \multicolumn{3}{|c|}{$0 \mathrm{~ms}$} & \multicolumn{3}{|c|}{$5000 \mathrm{~ms}$} \\
\hline & In & No & Out & In & No & Out & In & No & Out & In. & No & Out \\
\hline $\mathrm{MT}(\mathrm{ms})$ & 388 & 388 & 393 & 420 & 422 & 425 & 423 & 434 & 427 & 453 & 476 & 448 \\
\hline $\mathrm{CE}(\mathrm{mm})$ & -20.1 & -13.4 & -3.6 & -38.6 & -24.4 & -19.3 & -33.7 & -19.4 & $-8: 3$ & -52.2 & -37.7 & -17.1 \\
\hline $\mathrm{VE}(\mathrm{mm})$ & 25.1 & 21.2 & 23.4 & 27.3 & 27.3 & 30.0 & 20.1 & 20.8 & 20.5 & 30.1 & 26.7 & 32.0 \\
\hline
\end{tabular}




\section{Table 2}

Áveraỹo movomont time (MT), sonstant error (CE), and variable error (VE) in Experiment 2.

\begin{tabular}{|c|c|c|c|c|c|c|c|c|c|c|c|c|c|c|c|c|c|c|}
\hline \multirow{3}{*}{$\begin{array}{l}\text { Length: } \\
\text { Preparation: } \\
\text { Execution: }\end{array}$} & \multicolumn{9}{|c|}{$25 \mathrm{~cm}$} & \multicolumn{9}{|c|}{$35 \mathrm{~cm}$} \\
\hline & \multicolumn{3}{|c|}{$\ln$} & \multicolumn{3}{|c|}{ No } & \multicolumn{3}{|c|}{ Out } & \multicolumn{3}{|c|}{ In } & \multicolumn{3}{|c|}{ No } & \multicolumn{3}{|c|}{ Out } \\
\hline & $\ln$ & No & Out & In & No & Out & In & No & Out & In & No & Out & In & No & Out & In & No & Out \\
\hline MT (ms) & 386 & 394 & 399 & 405 & 391 & 402 & 388 & 398 & 393 & 446 & 446 & 438 & 438 & 446 & 436 & 435 & 429 & 432 \\
\hline $\mathrm{CE}(\mathrm{mm})$ & -4.2 & -1.0 & -0.7 & -3.2 & -1.2 & 0.6 & 0.7 & 2.0 & 3.5 & -6.9 & -5.7 & -3.4 & -6.9 & -6.9 & -2.6 & -4.3 & -2.7 & 0.3 \\
\hline $\mathrm{VE}(\mathrm{mm})$ & 5.5 & 6.3 & 9.6 & 5.9 & 5.4 & 6.4 & 6.8 & 5.8 & 6.3 & 6.0 & 6.5 & 7.1 & 6.4 & 6.4 & 7.0 & 6.3 & 6.9 & 7.0 \\
\hline
\end{tabular}


Table 3

Average movement time (MT), constant error (CE), and variable error (VE) in Experiment 3.

\begin{tabular}{|c|c|c|c|c|c|c|c|c|c|}
\hline \multirow{2}{*}{$\begin{array}{l}200 \mathrm{~ms} \\
\text { Preparation: } \\
\text { Execution: }\end{array}$} & \multicolumn{3}{|c|}{ In } & \multicolumn{3}{|c|}{ No } & \multicolumn{3}{|c|}{ Out } \\
\hline & $\ln$ & No & Out & $\ln$ & No & Out & In & No & Out \\
\hline MT (ms) & 404 & 406 & 411 & 399 & 416 & 414 & 406 & 406 & 406 \\
\hline $\mathrm{CE}(\mathrm{mm})$ & -3.9 & -2.1 & -2.0 & -2.2 & 0.5 & 1.0 & -1.9 & 0.9 & 1.3 \\
\hline $\mathrm{VE}(\mathrm{mm})$ & 6.7 & 7.6 & 6.7 & 7.9 & 7.5 & 7.0 & 6.8 & 6.9 & 7.2 \\
\hline \multirow{2}{*}{$\begin{array}{l}400 \mathrm{~ms} \\
\text { Preparation: } \\
\text { Execution: }\end{array}$} & \multicolumn{3}{|c|}{ In } & \multicolumn{3}{|c|}{ No } & \multicolumn{3}{|c|}{ Out } \\
\hline & $\ln$ & No & Out & $\ln$ & No & Out & In & No & Out \\
\hline $\mathrm{MT}(\mathrm{ms})$ & 458 & 454 & 457 & 459 & 470 & 456 & 467 & 450 & 453 \\
\hline $\mathrm{CE}(\mathrm{mm})$ & -4.3 & -0.9 & -0.2 & -2.3 & -1.0 & -0.4 & -2.2 & 0.5 & 1.5 \\
\hline VE $(\mathrm{mm})$ & 4.8 & 5.2 & 4.8 & 5.3 & 4.9 & 5.3 & 5.9 & 5.6 & 5.3 \\
\hline \multirow{2}{*}{$\begin{array}{l}600 \mathrm{~ms} \\
\text { Preparation: } \\
\text { Execution: }\end{array}$} & \multicolumn{3}{|c|}{ In } & \multicolumn{3}{|c|}{ No } & \multicolumn{3}{|c|}{ Out } \\
\hline & $\ln$ & No & Out & $\ln$ & No & Out & $\ln$ & No & Out \\
\hline $\mathrm{MT}(\mathrm{ms})$ & 494 & 493 & 488 & 499 & 490 & 490 & 496 & 487 & 484 \\
\hline $\mathrm{CE}(\mathrm{mm})$ & -5.2 & -1.5 & -1.7 & -3.2 & -0.7 & -1.8 & -2.9 & -0.6 & -0.9 \\
\hline VE $(\mathrm{mm})$ & 4.7 & 5.3 & 5.0 & 4.5 & 5.4 & 5.8 & 4.9 & 5.1 & 4.9 \\
\hline
\end{tabular}




\section{LIST OF FIGURES}

\section{Experiment 1}

Figure 1. Constant error ( $\mathrm{mm})$ as a function of Configuration, Length, and

Delay. Note the absence of a Configuration by Delay interaction.

Figure 2. Spatial variability $(\mathrm{mm})$ as a function of Kinematic Marker and Length.

Figure 3. Spatial variability $(\mathrm{mm})$ as a function of Kinematic Marker and Delay.

Figure 4. Difference score $(\mathrm{mm})$ as a function of Configuration.

Figure 5. Difference score $(\mathrm{mm})$ as a function of Kinematic Marker and Configuration.

\section{Experiment 2}

Figure 6. Constant error $(\mathrm{mm})$ as a function of Preparation Configuration and Execution Configuration.

Figure 7. Spatial variability (mm) as a function of Kinematic Marker.

Figure 8. Difference score $(\mathrm{mm})$ as a function of Condition.

Figure 9. Difference score ( $\mathrm{mm})$ as a function of Kinematic Marker and

Condition. Note the difference between movements prepared with tails-in versus tails-out stimuli.

Figure 10. Difference score ( $\mathrm{mm})$ as a function of Kinematic Marker and

Condition. Note the difference between movements executed with tails-in versus tails-out stimuli.

\section{Experiment 3}

Figure 11. Constant error (mm) as a function of Preparation Configuration and Execution Configuration.

Figure 12.. Spatial variability $(\mathrm{mm})$ as a function of Kinematic Marker.

Figure 15. Difference score $(\mathrm{mm})$ as a function of Condition. 
Figure 14 Difference score $(\mathrm{mm})$ as a function of Kinematic Marker and

Condition. Note the difference between movements prepared with tails-in versus tails-out stimuli.

Figure 15. Difference score $(\mathrm{mm})$ as a function of Kinematic Marker and

Condition. Note the difference between movements executed with tails-in versus tails-out stimuli.

Figure 16. Difference score $(\mathrm{mm})$ as a function of Kinematic Marker and

Condition with $200 \mathrm{~ms}$ of visual feedback. Note the difference between movements prepared with tails-in versus tails-out stimuli.

Figure 17. Difference score $(\mathrm{mm})$ as a function of Kinematic Marker and

Condition with $400 \mathrm{~ms}$ of visual feedback. Note the difference between movements prepared with tails-in versus tails-out stimuli.

Figure 18. Difference score (mm) as a function of Kinematic Marker and

Condition with $600 \mathrm{~ms}$ of visual feedback. Note the difference between movements prepared with tails-in versus tails-out stimuli.

Figure 19. Difference score ( $\mathrm{mm}$ ) as a function of Kinematic Marker and

Condition with $200 \mathrm{~ms}$ of visual feedback. Note the difference between movements executed with tails-in versus tails-out stimuli.

Figure 20. Difference score ( $\mathrm{mm})$ as a function of Kinematic Marker and Condition with $400 \mathrm{~ms}$ of visual feedback. Note the difference between movements executed with tails-in versus tails-out stimuli.

Figure 21. Difference score $(\mathrm{mm})$ as a function of Kinematic Marker and Condition with $600 \mathrm{~ms}$ of visual feedback. Note the difference between movements executed with tails-in versus tails-out stimuli. 


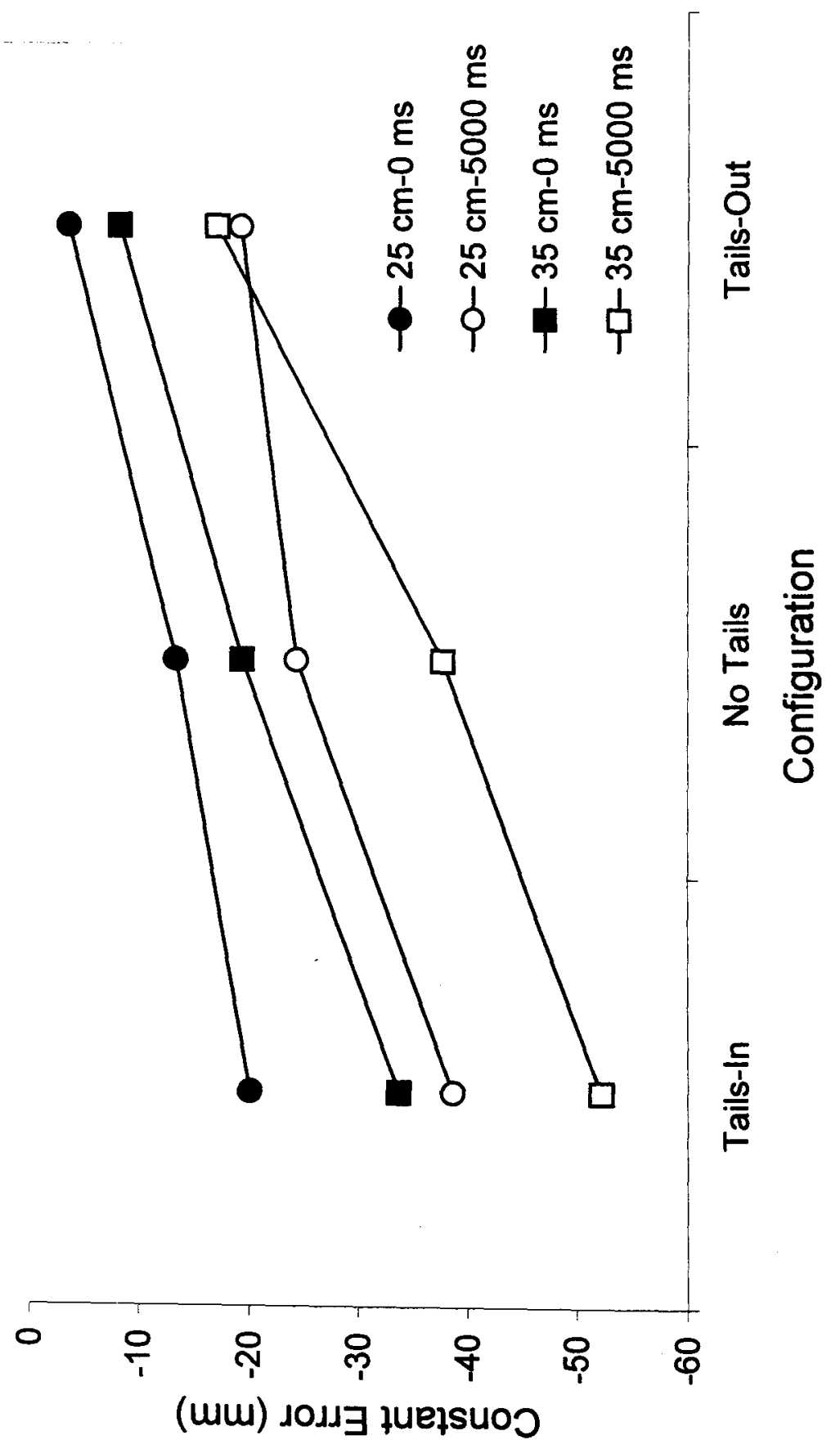




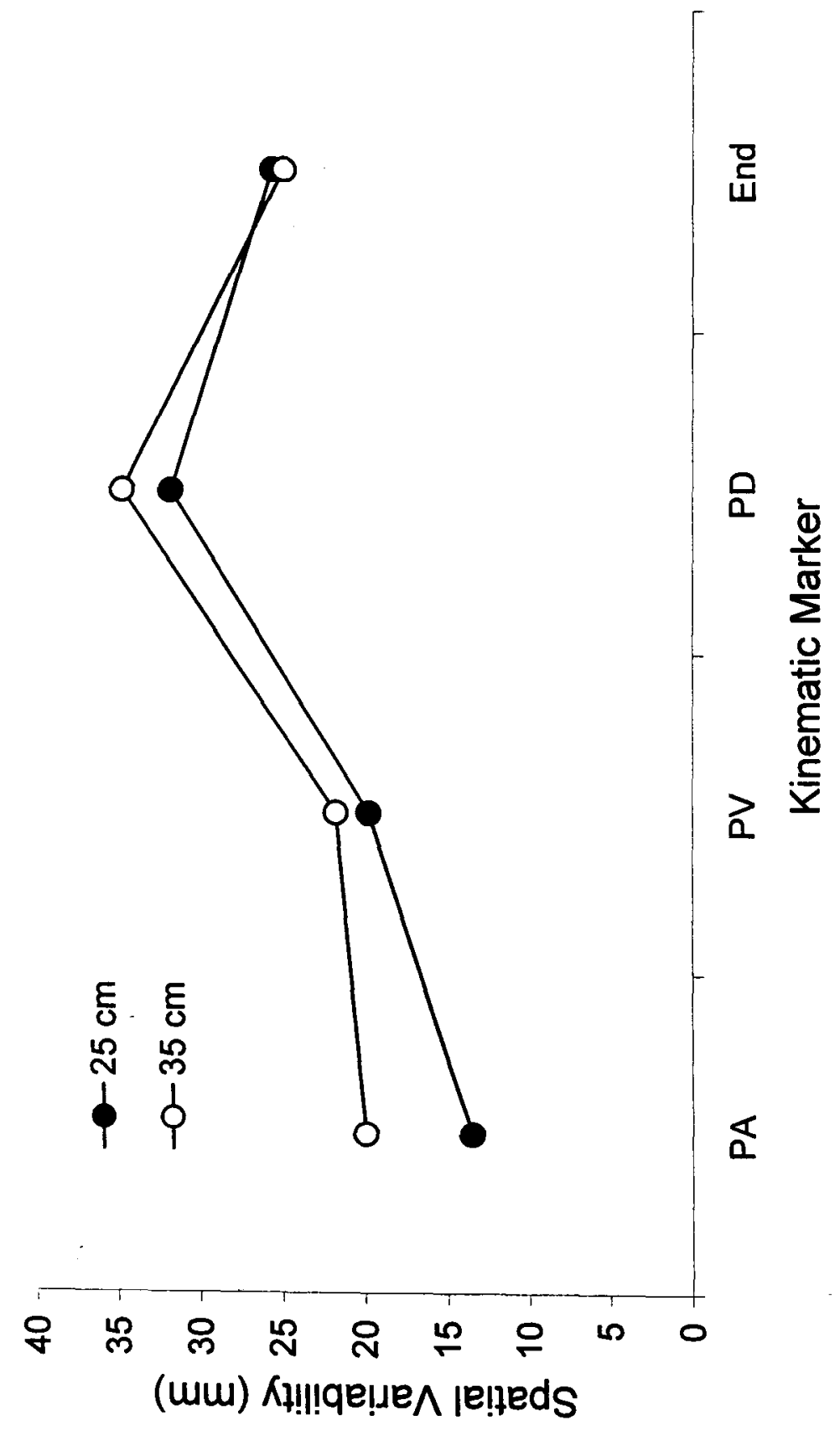




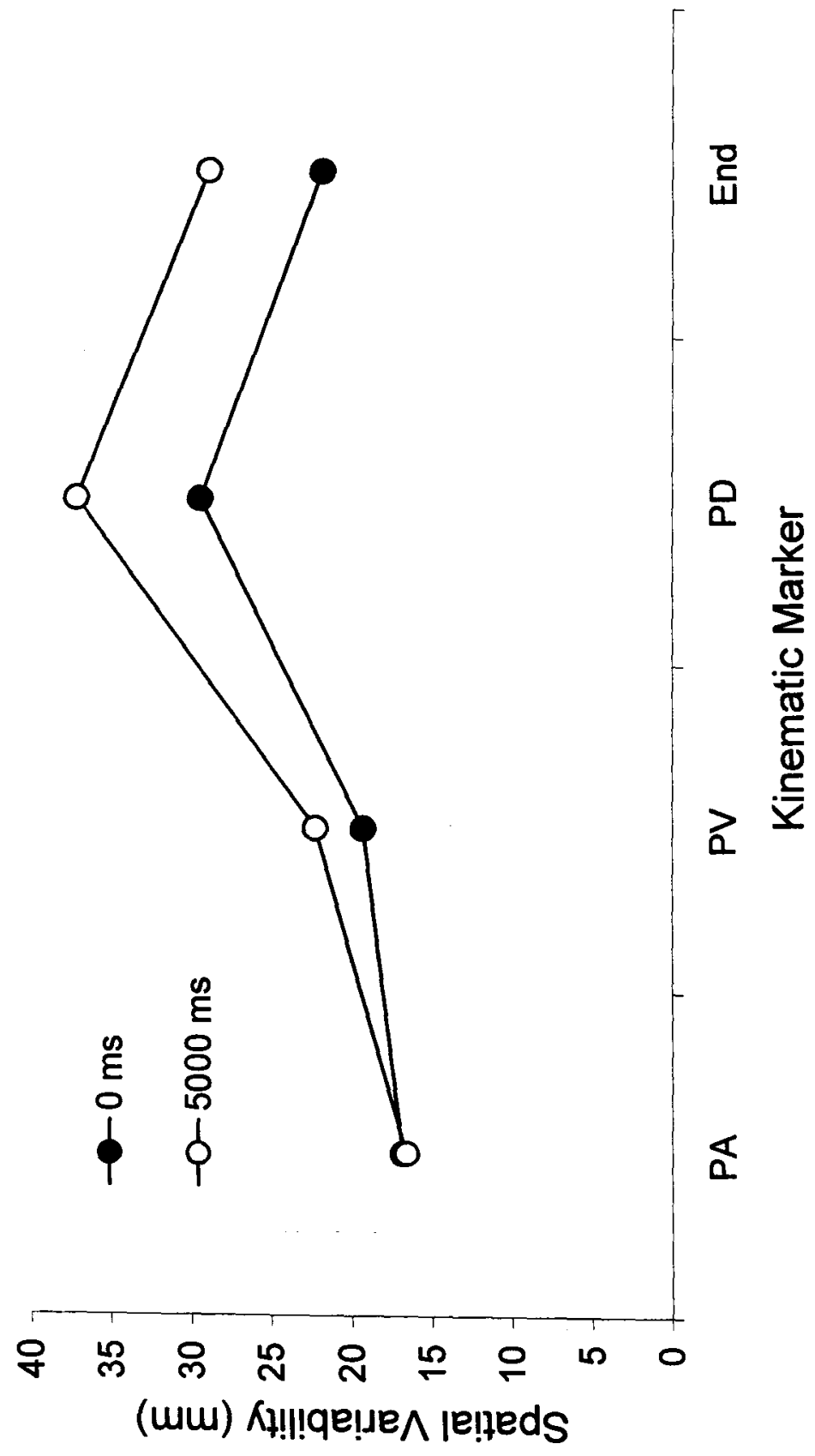




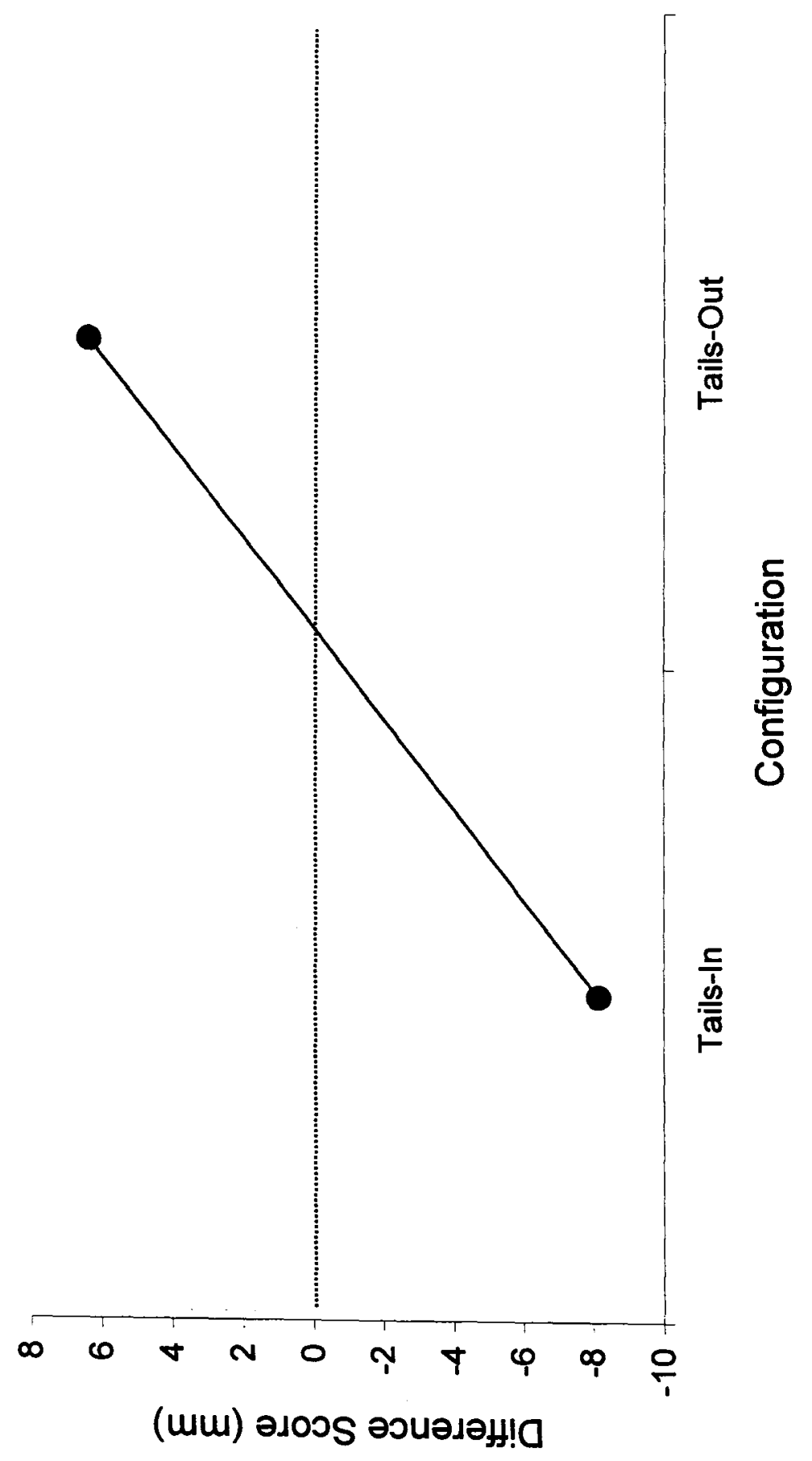




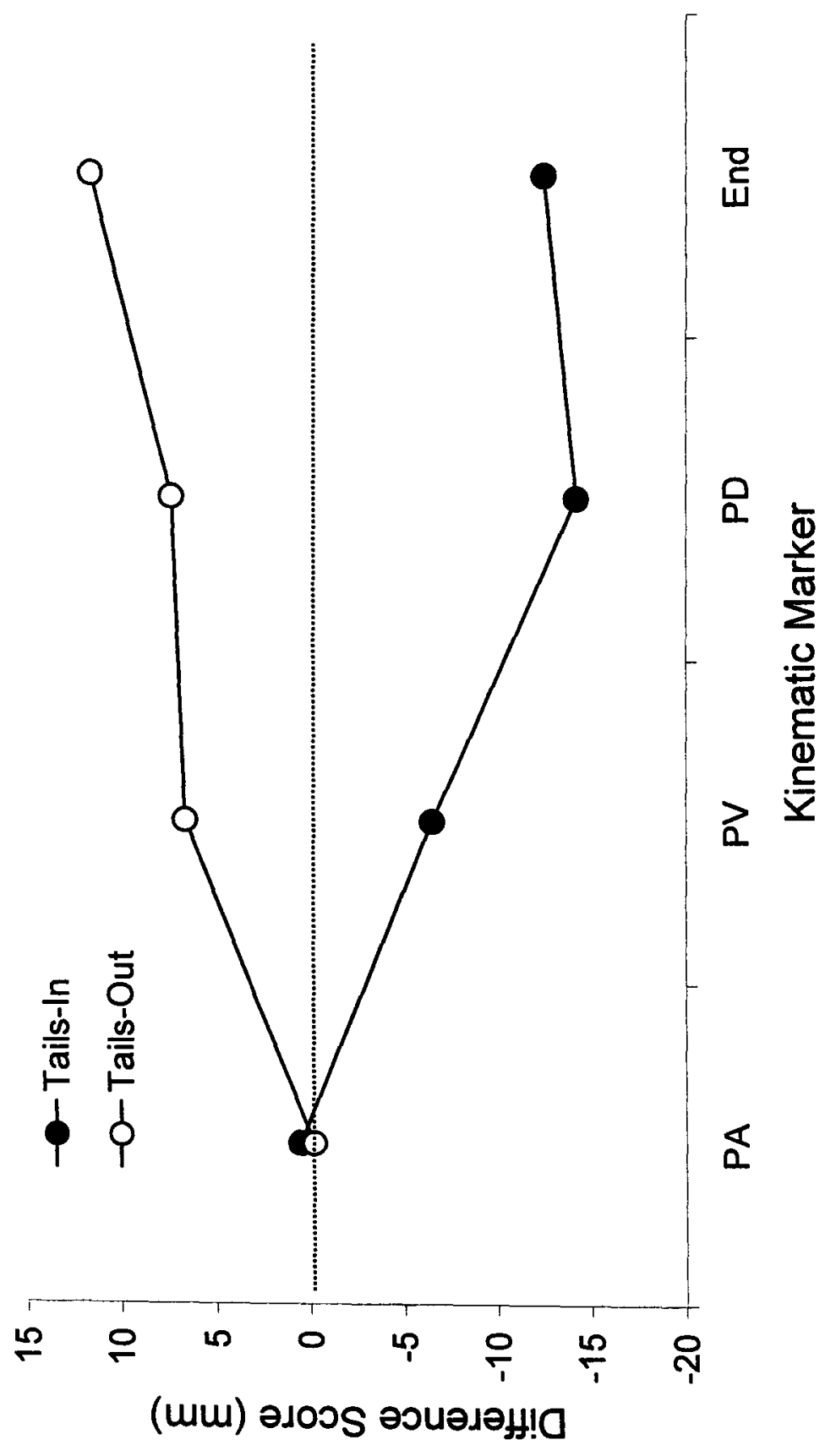




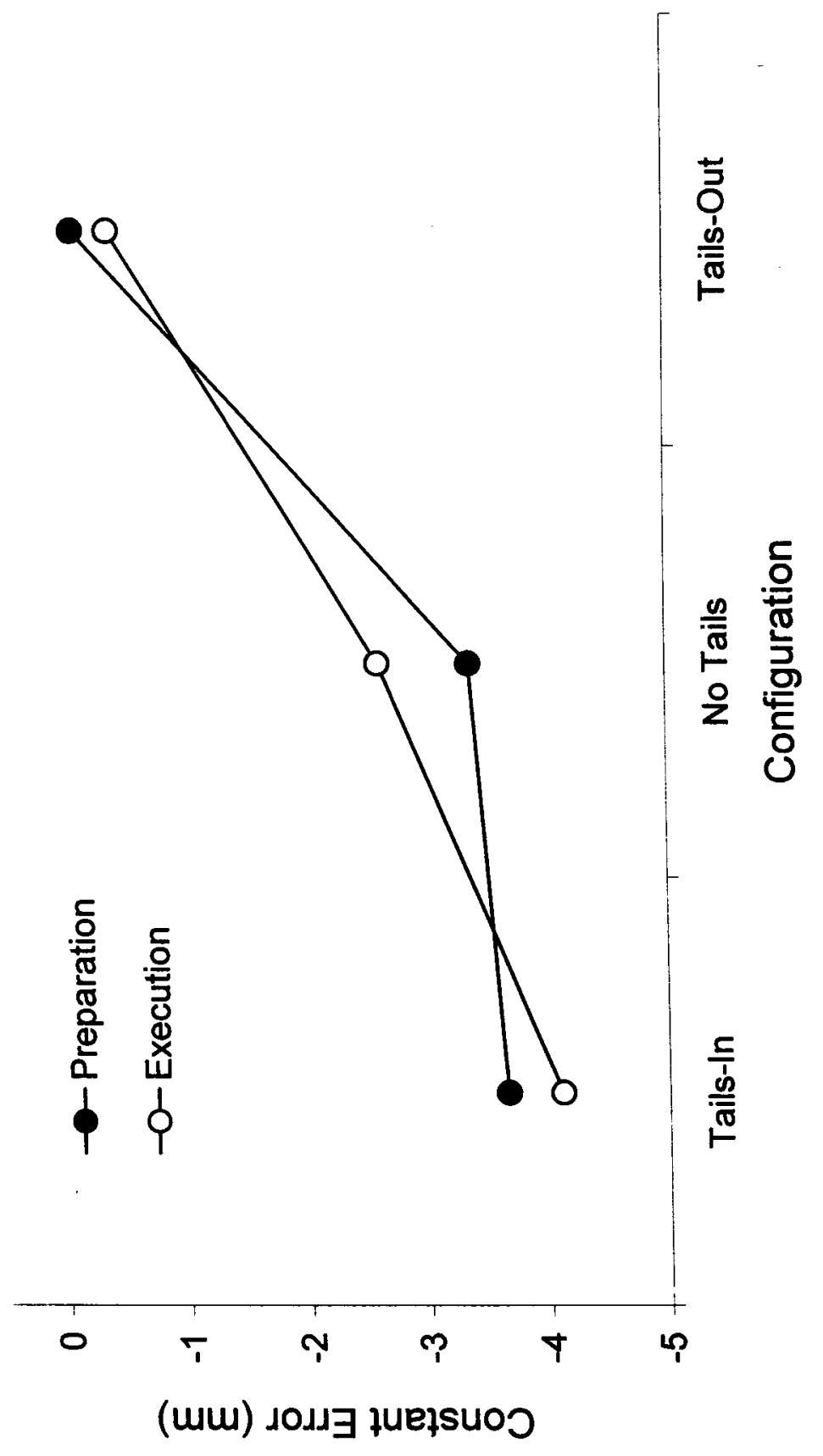




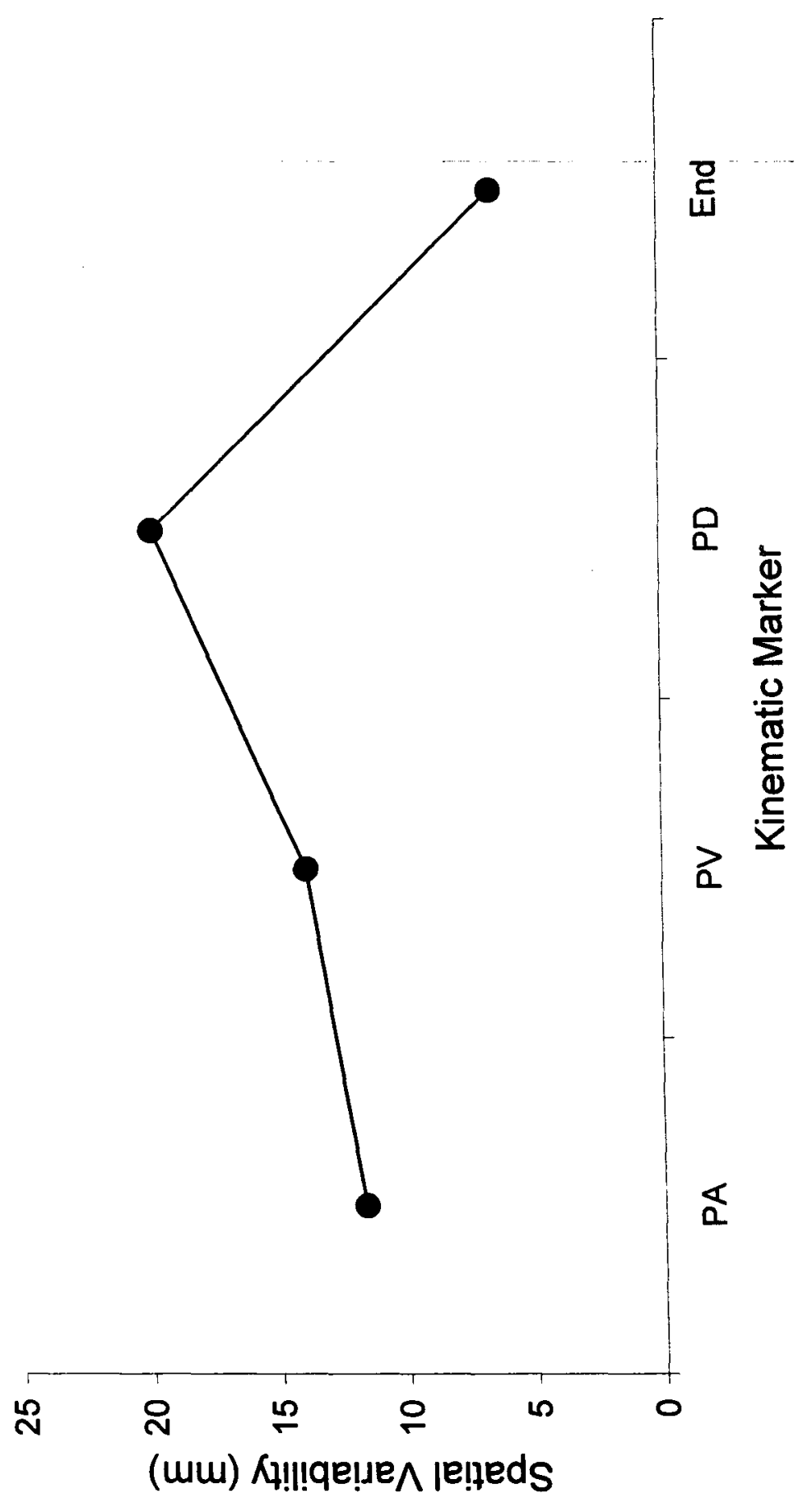




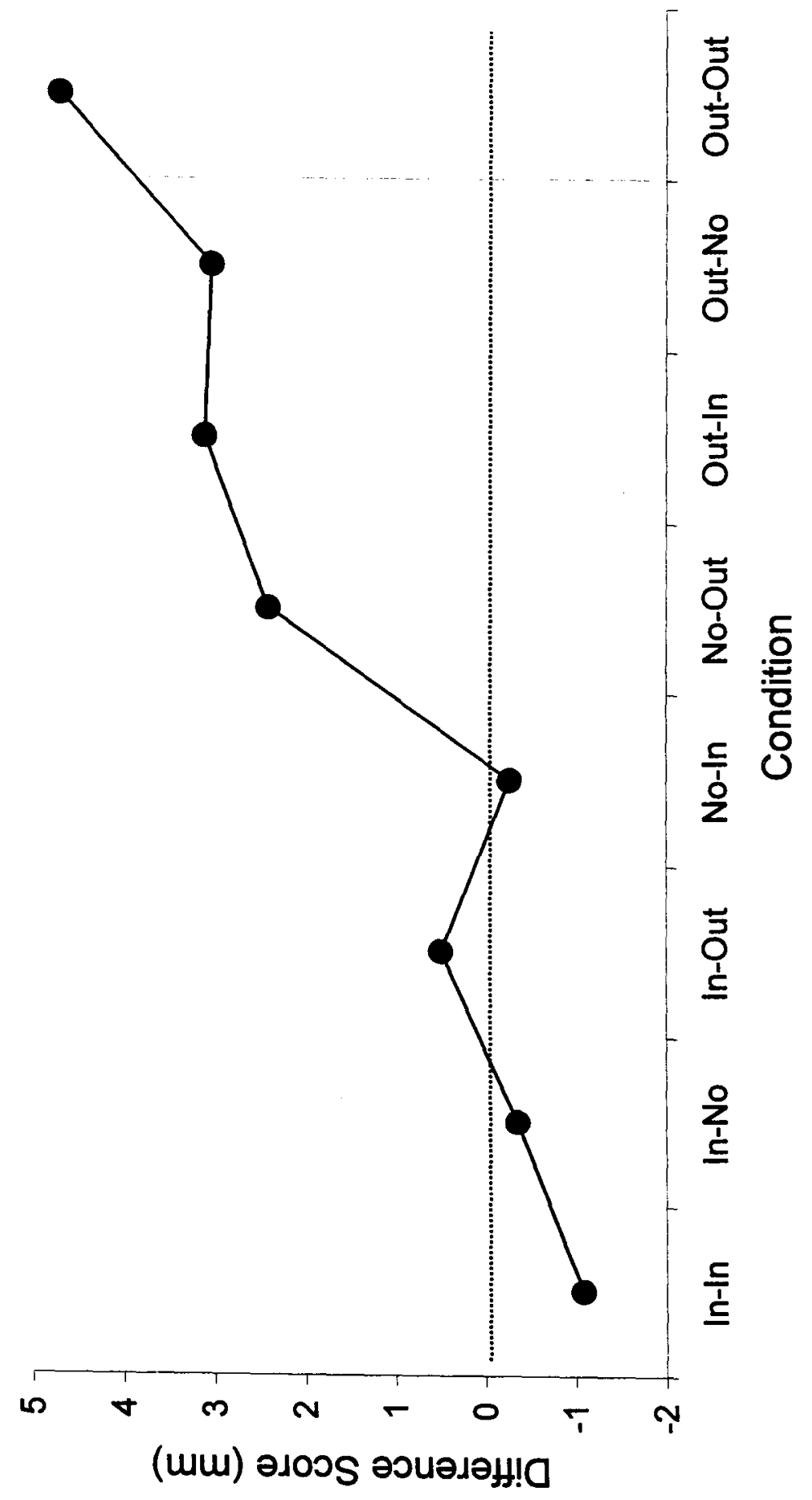



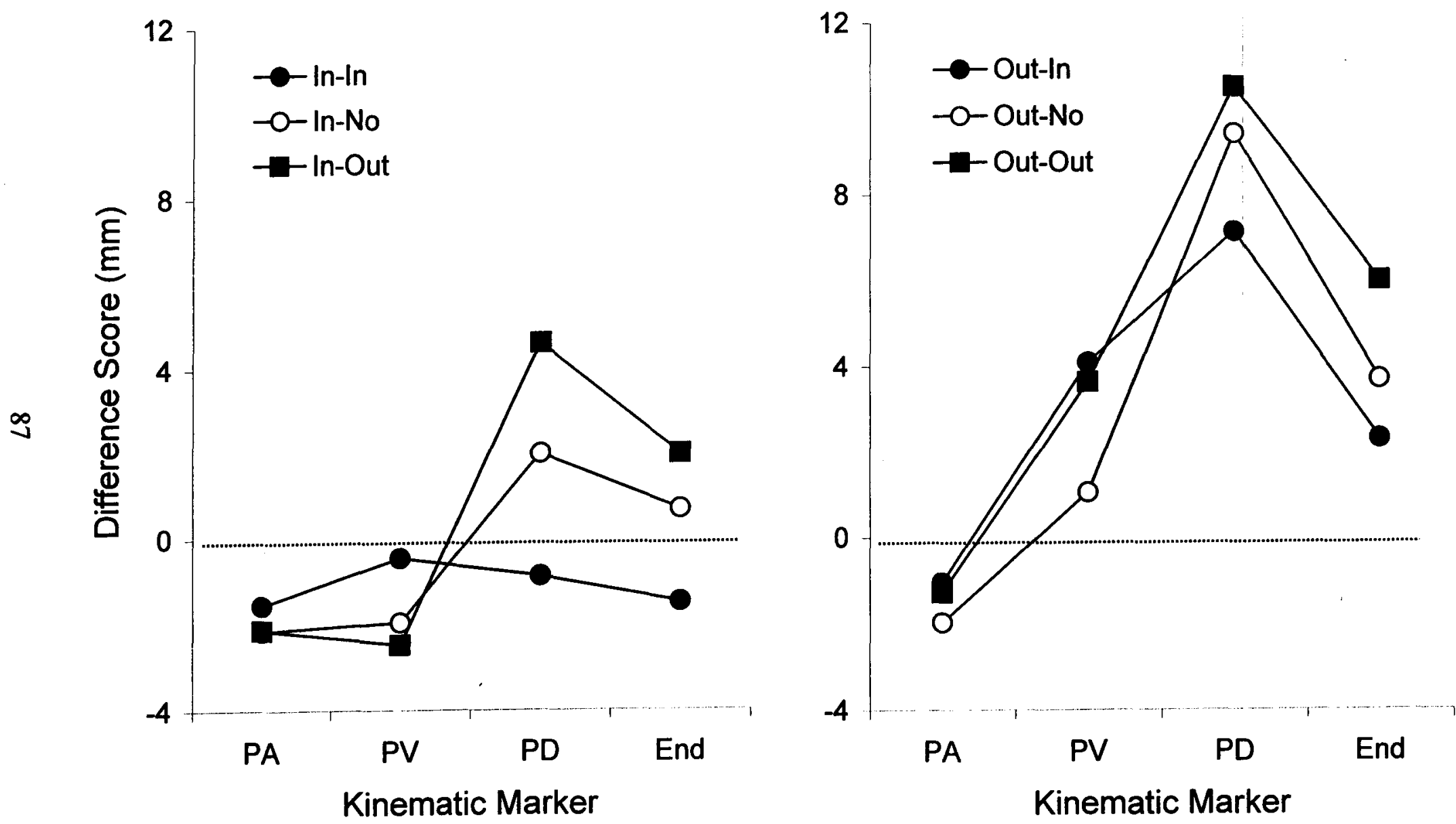

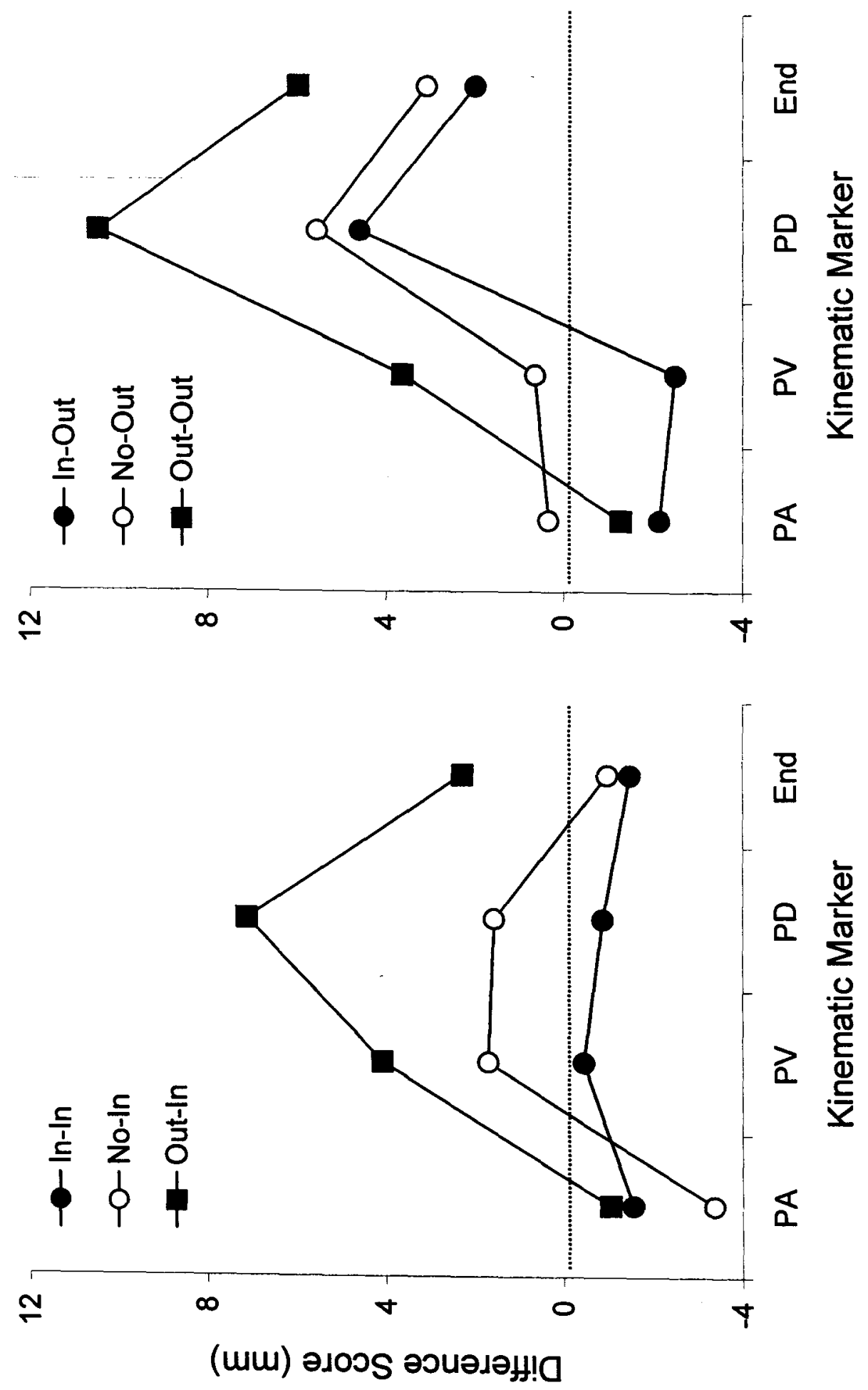


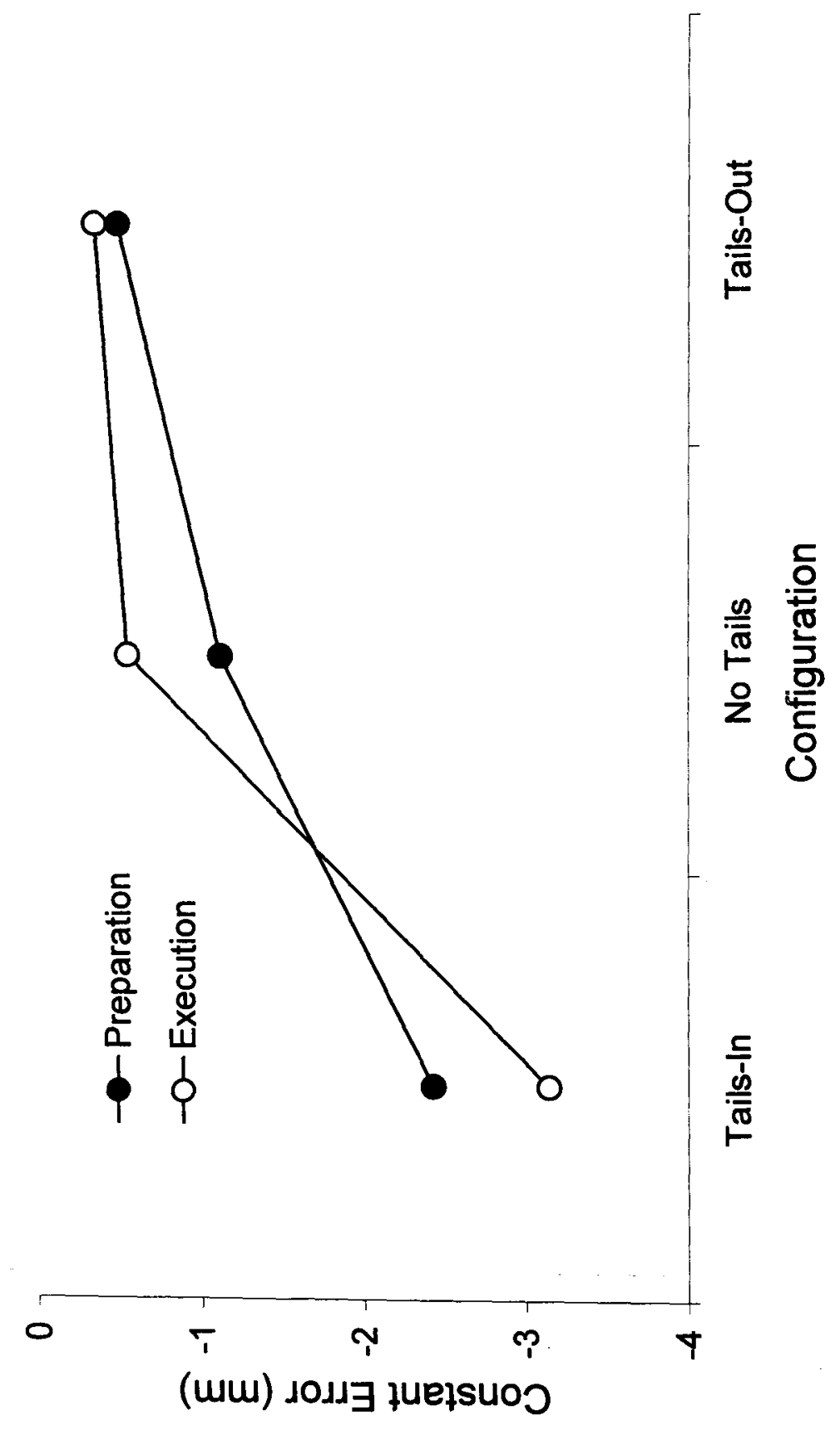




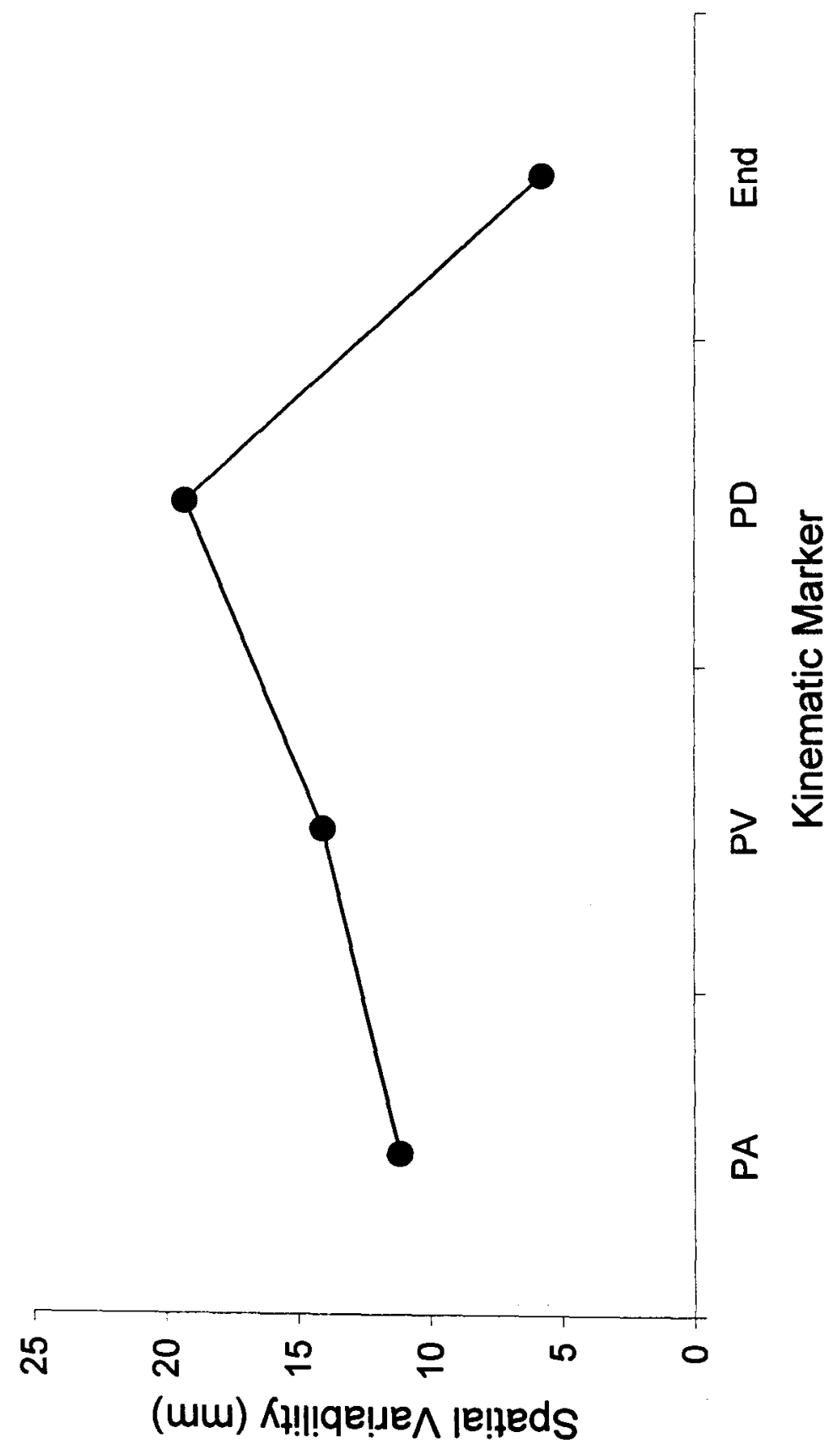




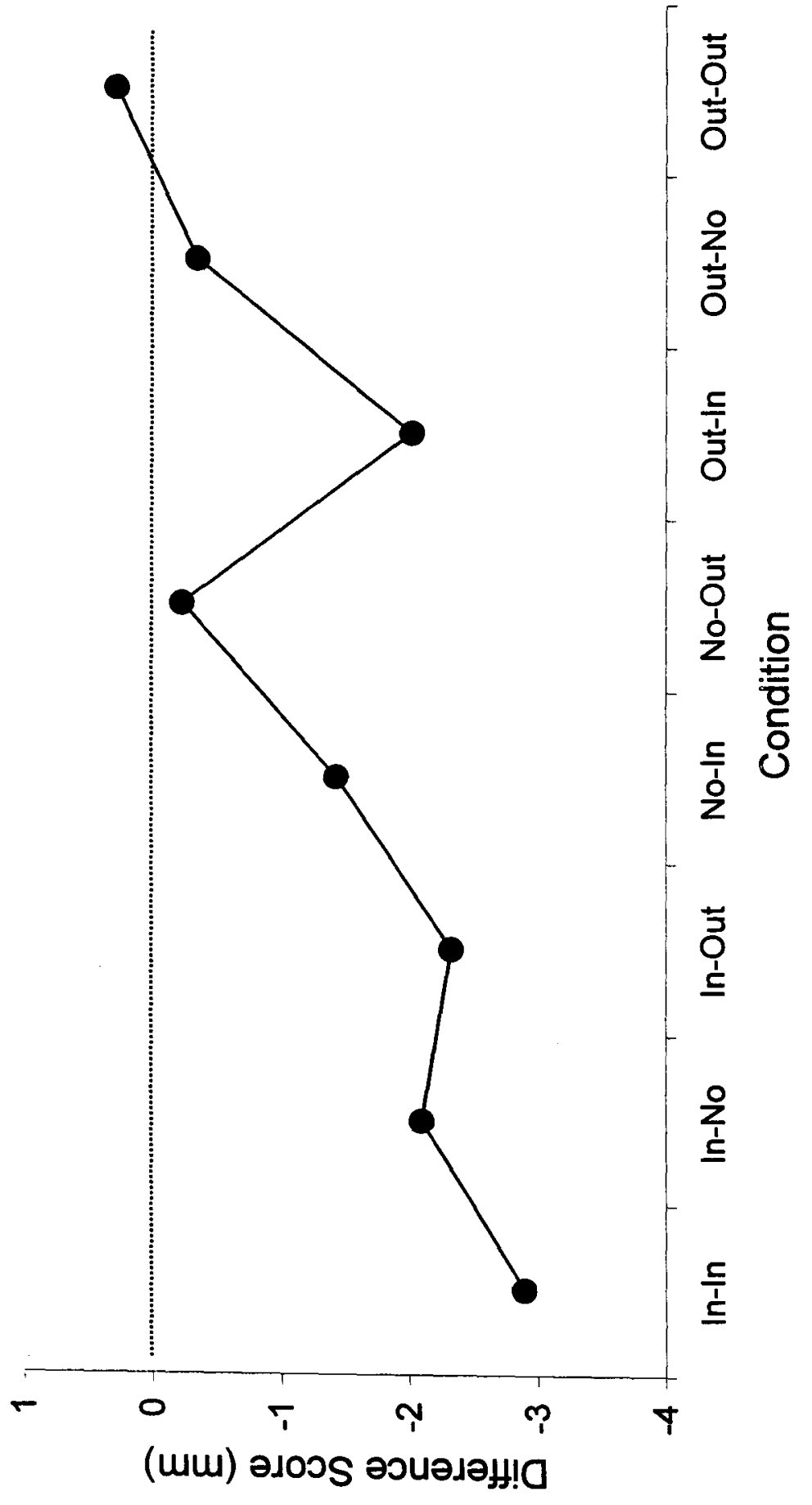



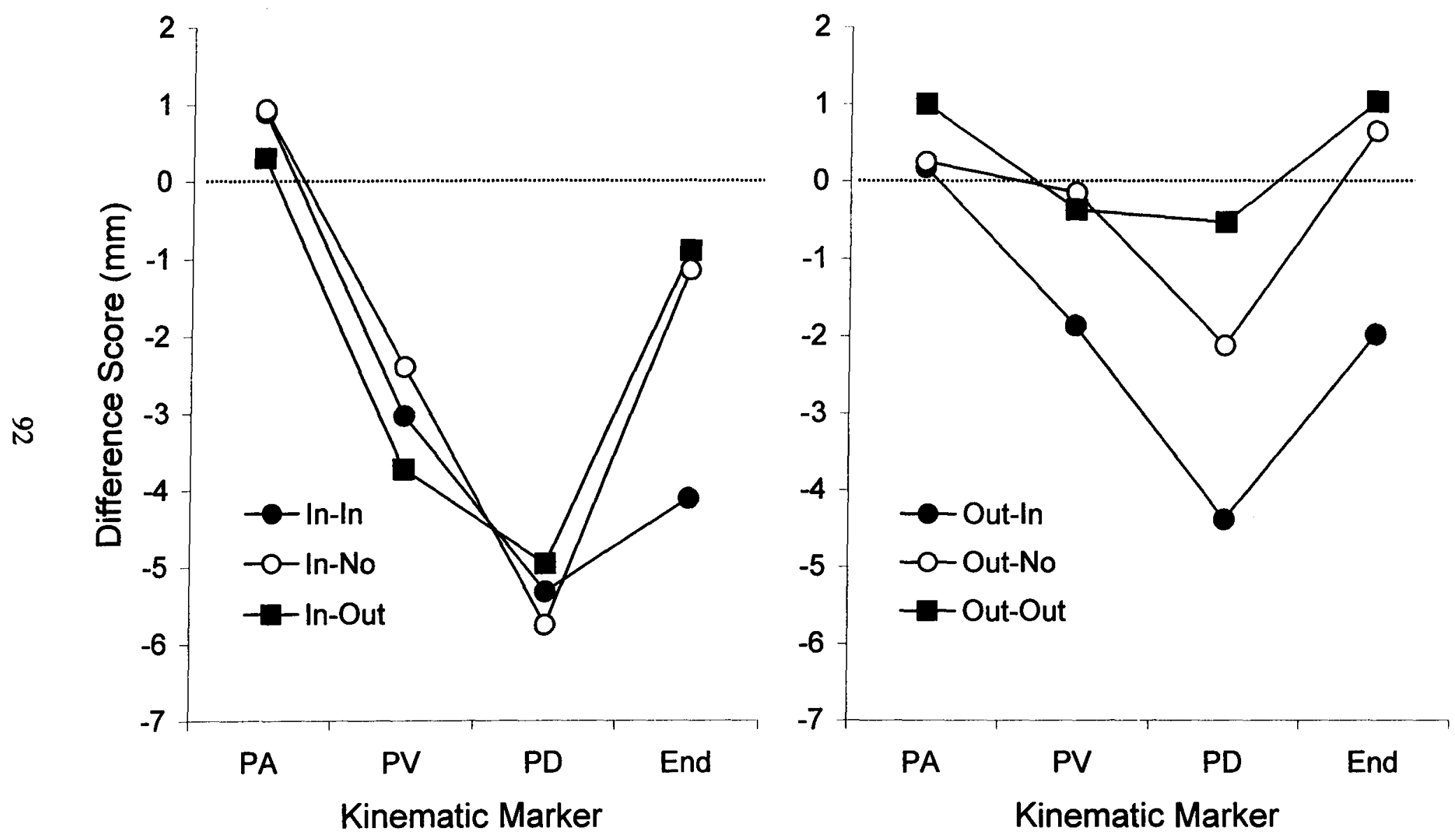

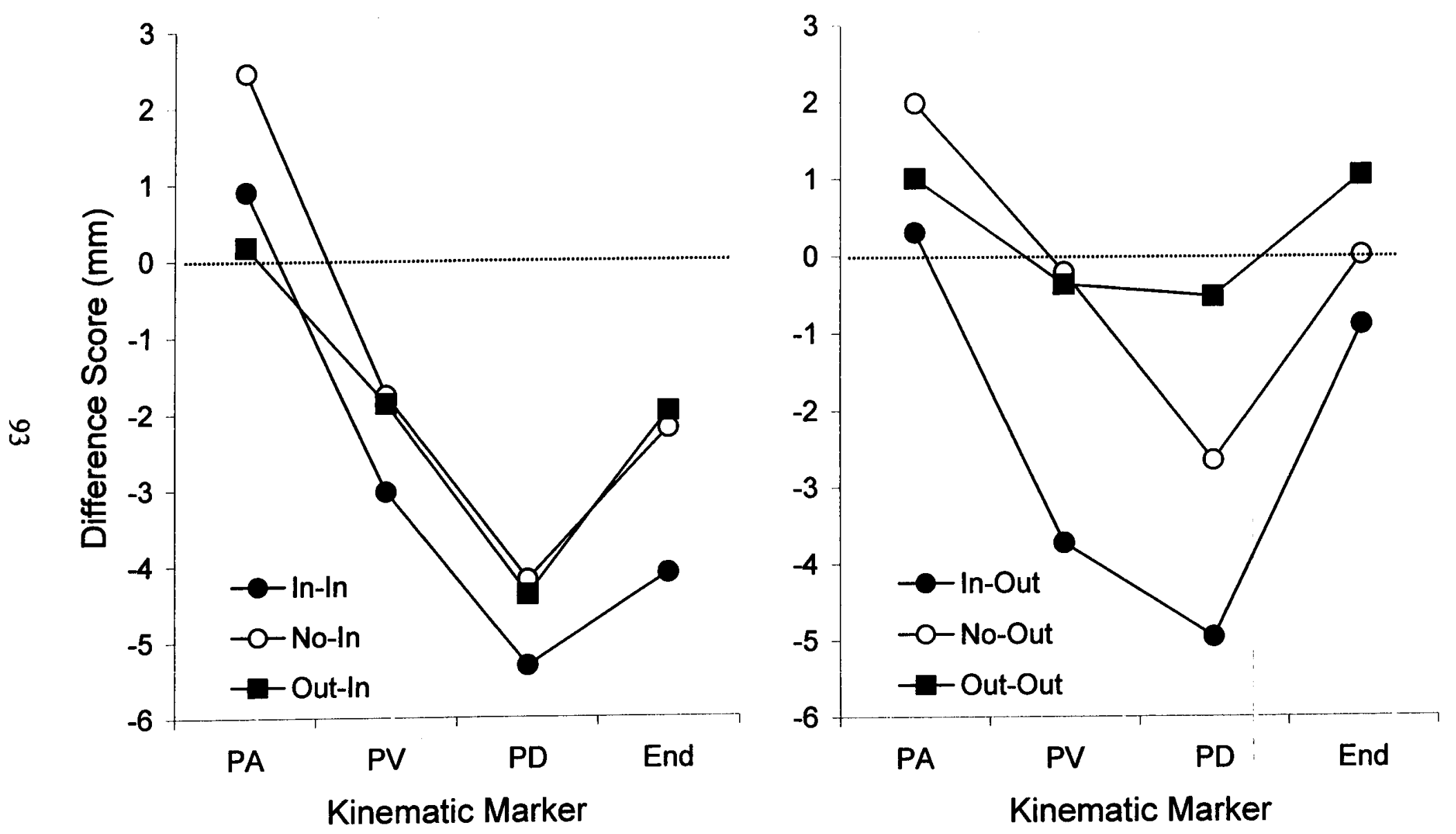

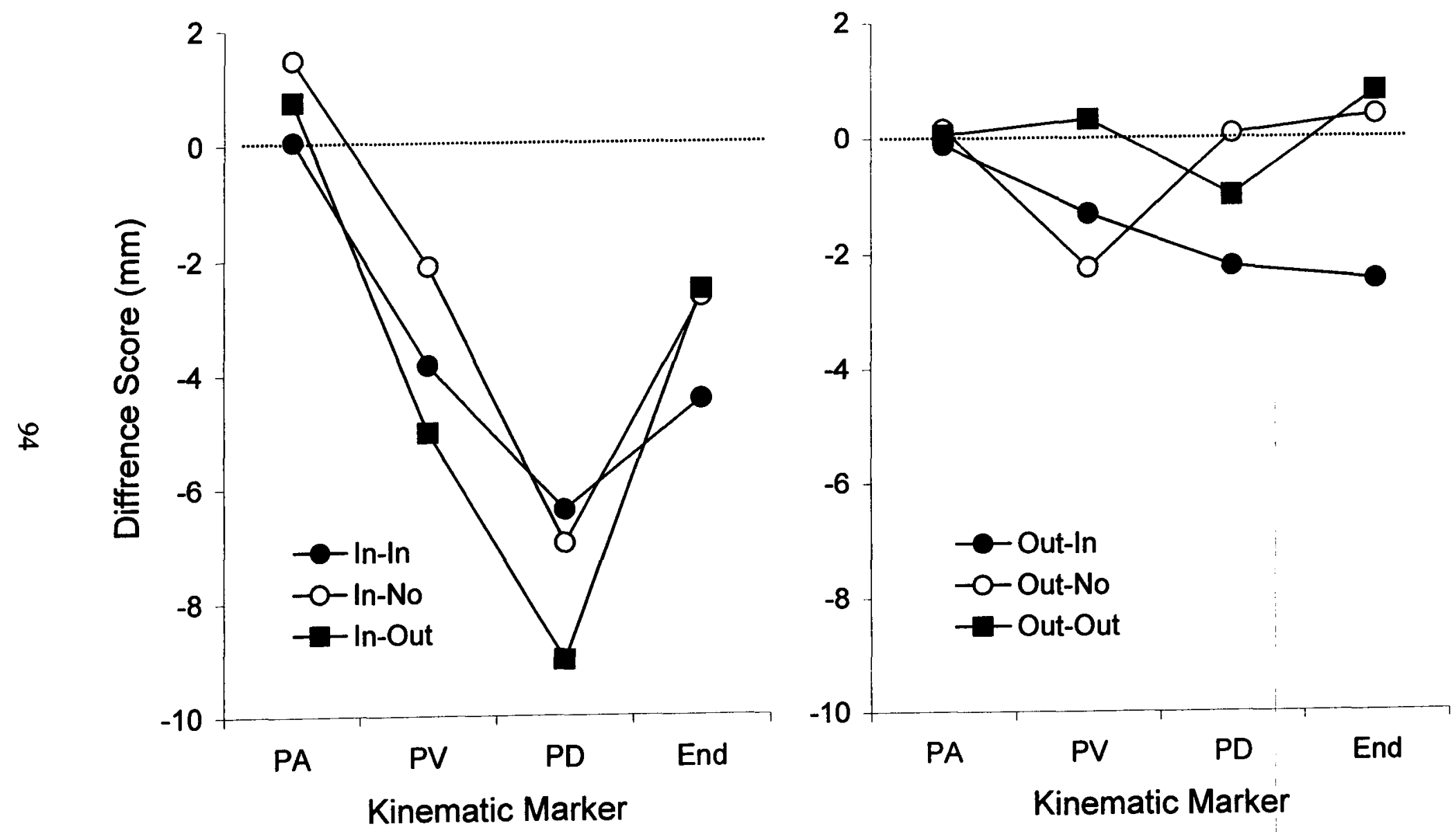

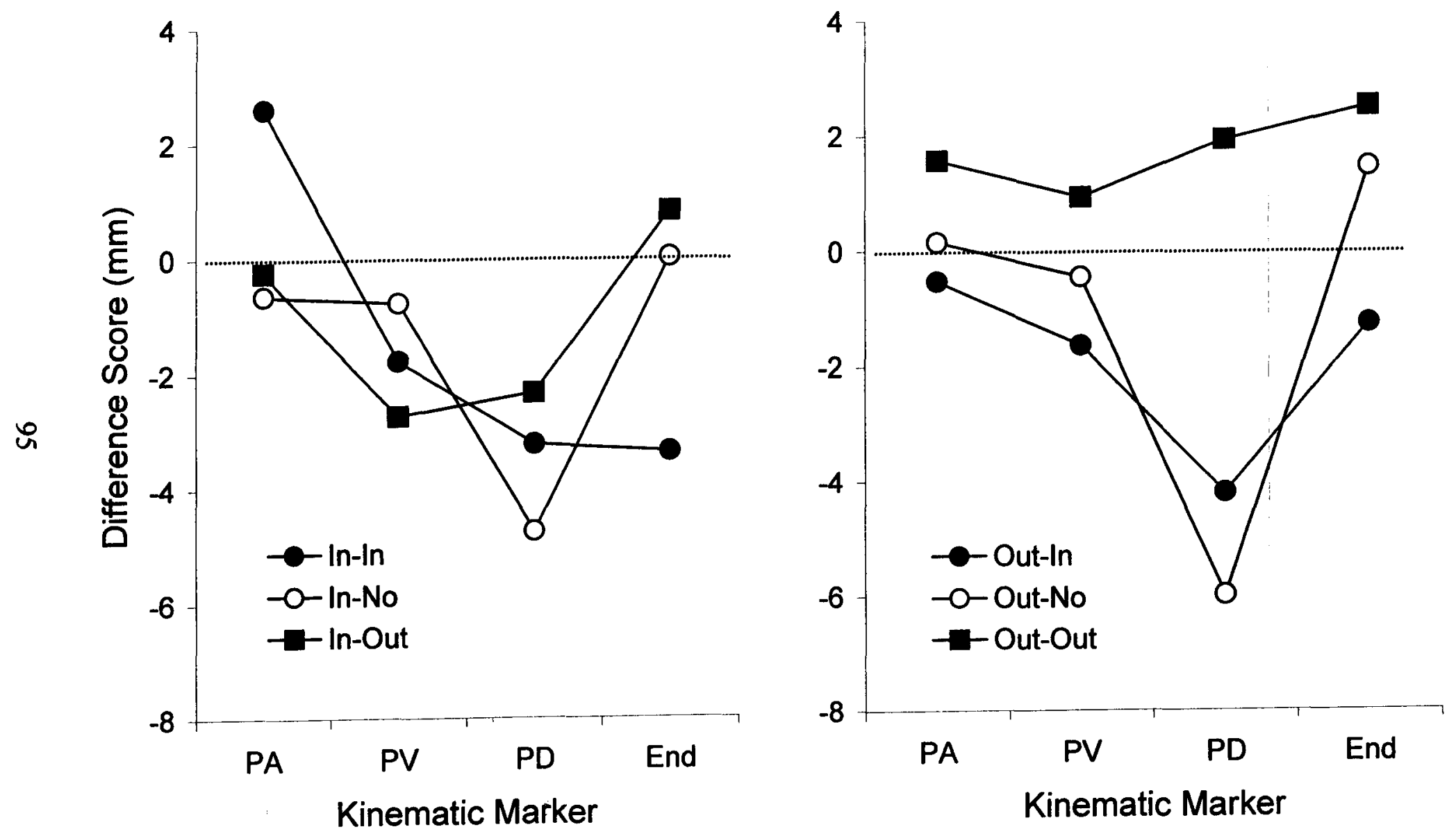

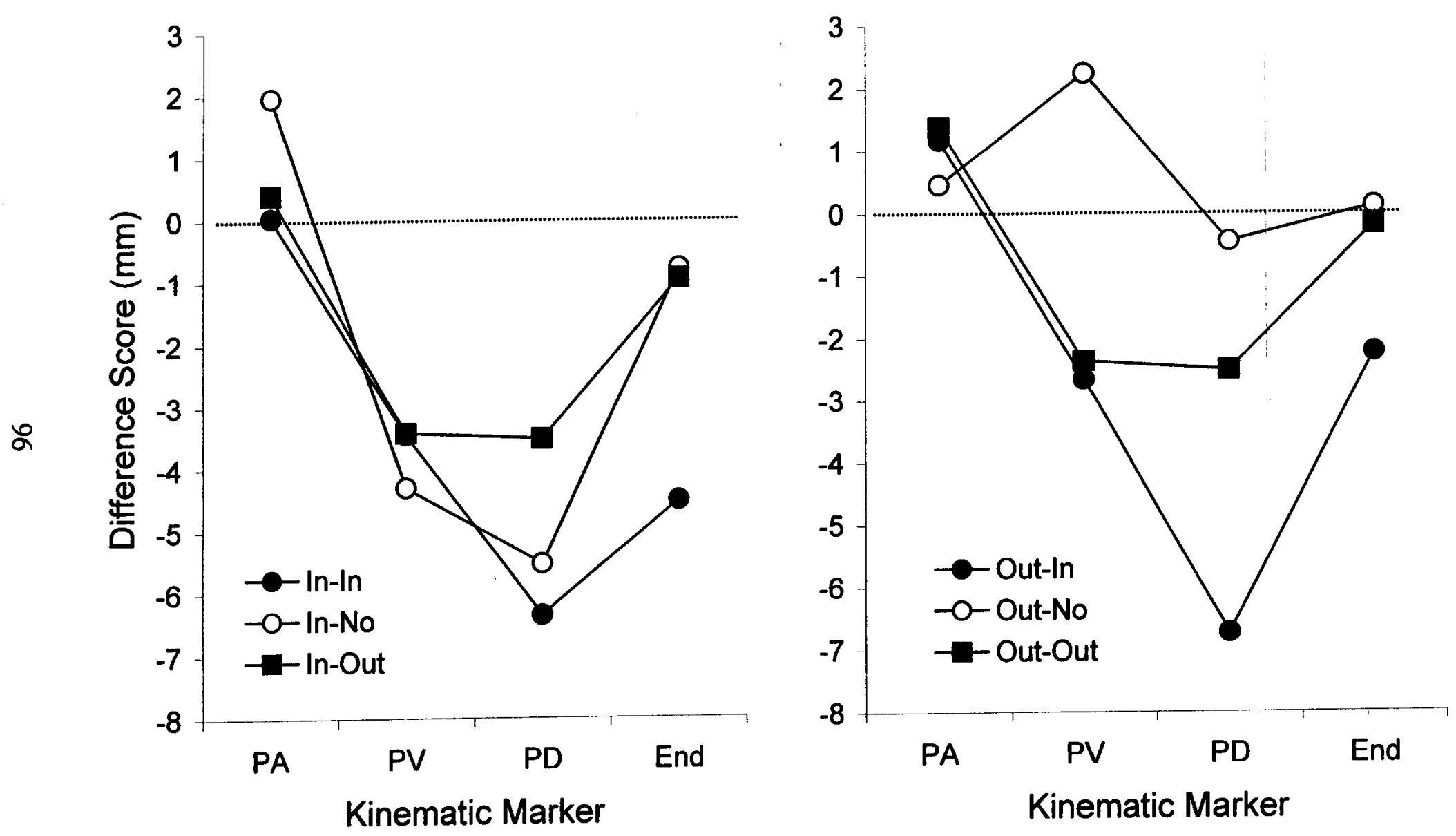

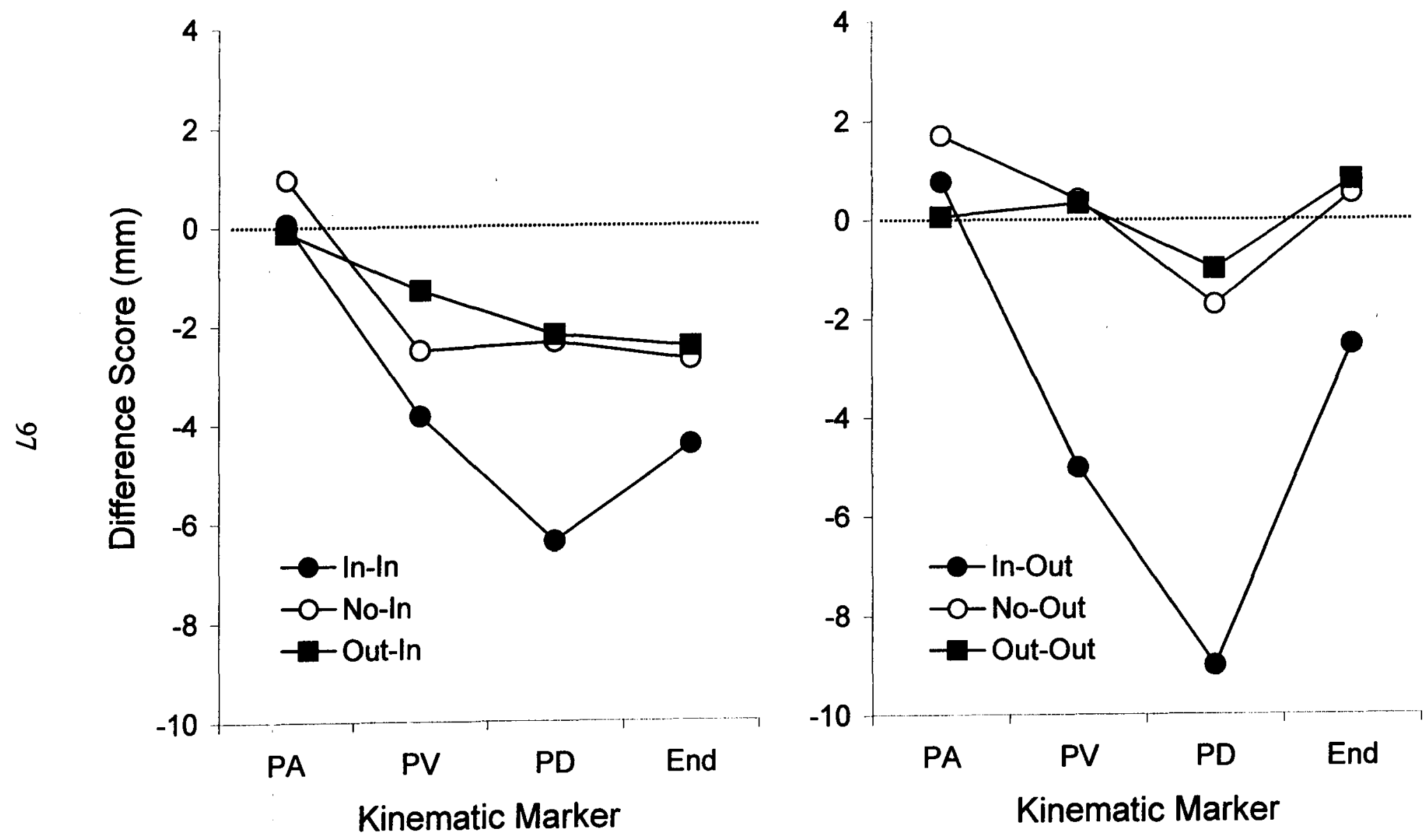

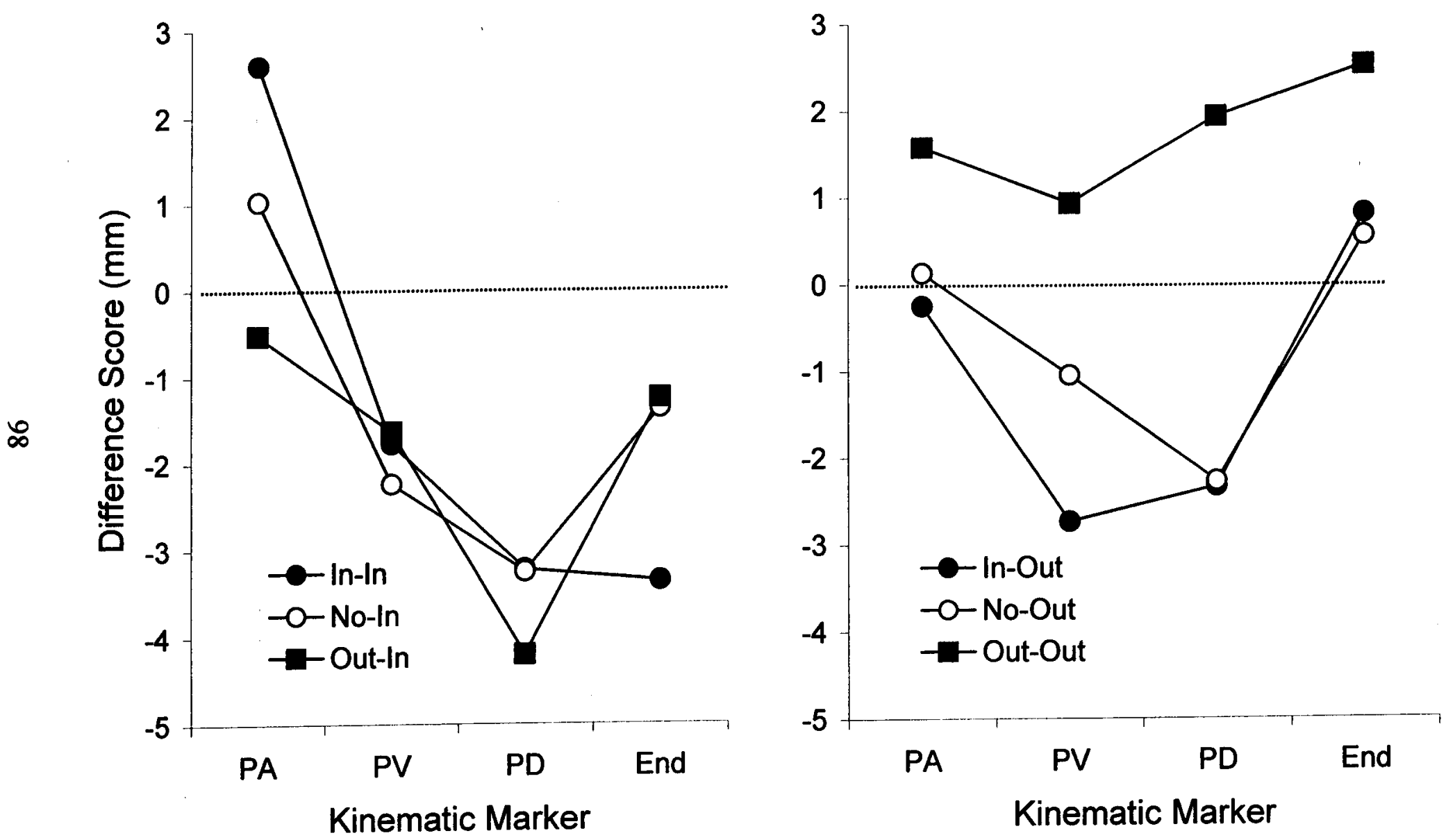

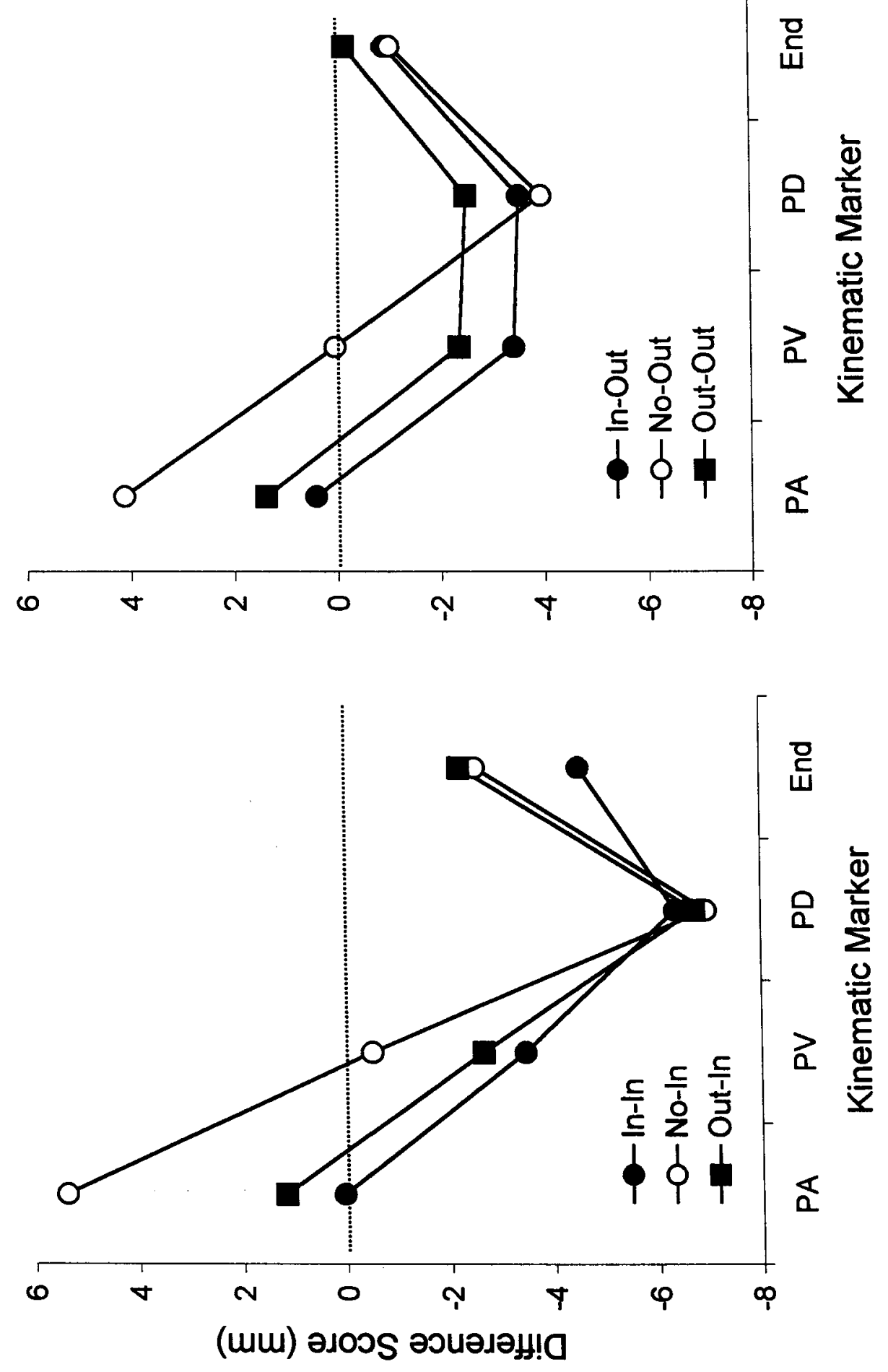